\title{
ANALYTIC SOLUTIONS AND PARTICLE SIMULATIONS OF CROSS-FIELD PLASMA SHEATHS
}

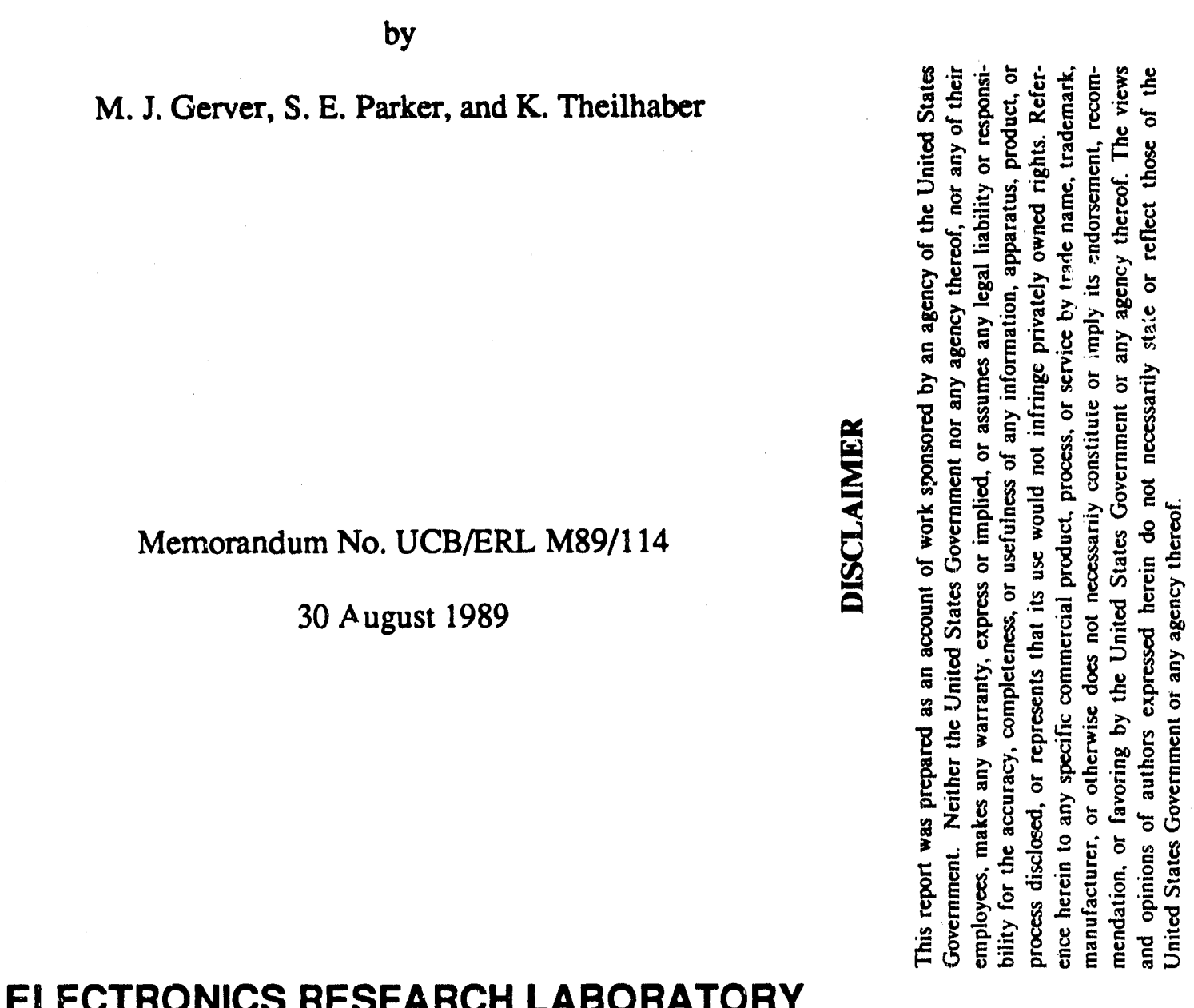

ELECTRONICS RESEARCH LABORATORY

College of Engineering

University of California, Berkeley

94720 


\title{
Analytic Solutions and Particle Simulations of \\ Cross-Field Plasma Sheaths
}

\author{
M. J. Gerver \\ Plasma Fusion Center, Massachusetts Institute of Technology, Cambridge, MA 02139
}

S. E. Parker and K. Theilhaber*

Electronics Research Laboratory, University of California, Berkeley, CA 94720

Particles simulations have been made of an infinite plasma slab, bounded by absorbing conducting walls, with a magnetic field parallel to the walls. The simulations have been either 1-D or 2-D, with the magnetic field normal to the simulation plane. Initislly, the plasma has a uniform density between the walls, and there is a uniform source of ions and electrons to replace particles lost to the walls. In the 1-D case, there is no diffusion of the particle guiding centers, and the plasma remains uniform in density and potential over most of the slab, with sheaths about a Debye length wide where the potential rises to the wall potential. In the 2-D case, the density profile becomes parabolic, going almost to zero at the walls, and there is a quasineutral presheath in the bulk of the plasma, in addition to sheaths near the walls. Analytic expressions are found for the density and potential profiles in both cases, including, in the 2-D case, the magnetic presheath due to finite ion Larmor radius, and the effects of the guiding center diffusion rate being either much less than or much greater than the energy diffusion rate. These analytic expressions are shown to agree with the simulations. A 1-D simulation with Monte Carlo guiding center diffusion included gives results that are in good agreement with the much more expensive 2-D simulation.

* Present address: Lawrence Livermore National Laboratory, University of California, Livermore, CA 94550 


\section{Introduction}

Particle simulations can be a useful trol for studying the formation and steady state behavior of sheaths near the boundaries of plasmas. To be certain of including all relevant physics, such simulations would have to be fully three-dimensional, but 3-D simulations are very expensive, and it is completely impractical to make extensive sets of 3-D simulations with varying parameters. Even with 2-D simulations, large numbers of runs can be impractical, especially if the phenomena being simulated depend on resonances involving only a small class of particles, or on the interraction of physics on different time scales, since in these cases a large number of particles will be needed to reduce noise to a low level, or a large nurnber of time steps will be needed. One-dimensional simulations, on the other hand, are very cheap, and it is easy to make many runs with many time steps and particles, but 1-D simulations oflen leave out important physics that is present in two or three dimensions. If it is possible to modify a 1-D simulation in such a way that the essential two or three dimensional physics can be included, without doing a fully two dimensional run, then a great deal of computer time can be saved. At the very least, 1-D runs can then be used to explore parameter space, and to determine an optimum set of parameters for making a few 2-D runs, which may be used to verify that the two-dimensional physics is being treated correctly in the 1-D runs.

To illustrate this, we show, in Fig. 1, the potential profile $\Phi(x)$ and electron density profile $n_{\mathrm{e}}(x)$ for a 1-D simulation and, in Fig. 2, for a 2-D simulation, of a plasma slab bounded by floating conducting walls at $x= \pm R_{p}$, with a uniform magnetic field $B_{0}$ in the $z$ direction, parallel to the walls and perpendicular to the simulation space. Models related to this one can be used to describe the interface between the closed field-line region and the scrape-off layer in tokamaks. ${ }^{1}$

The simulations were done using the electrostatic two-dimensional particle simulation code ES2. ${ }^{2}$ This code is of the explicit type, in that it includes full electron and ion dynamics, and simulates plasma behavior on the time scale of the electron dynamics. ${ }^{3}$ The simulation plane is periodic in the $y$ directinn with periodicity $L_{y}$, and is bounded at $x=R_{p}$ by a floating, perfectly conduciing wall, which absorbs all particles. Inversion symmetry is imposed at $x=0$ (so that in effect there is another wall at $x=-R_{p}$ ), as well as the boundary condition $\Phi=0$. A uniform ionization source is simulated by creating ion-electron pairs at ranciom positions and times, and with random and uncorrelated initial velocities (with a maxwellian distribution). For the $1-\mathrm{D}$ simulation, $L_{y}$ was set equal to 
0 , eliminating the $y$ dimension. In both simulations, the mass ratio $m_{i} / m_{e}$ was 40 , the ion and electron temperatures were initially equal (and remained about equal) the thermal ion Larmor radius was about $0.2 R_{p}$, and the thermal electron Larmor radius was about $0.03 R_{p}$. In the 1-D simulation, about 6000 particles of each species were present at a given time, and the Debye length was $0.03 R_{p}$. For the $2-\mathrm{D}$ simulation, about 25000 particles of each species were present at a given time, in a simulation area of $4 R_{p}^{2}$, and the Debye length was $0.06 R_{p}$. The 2-D simulation was run for 1000 ion gyroperiods, at which time it kad reached a fairly steady state. (The 1-D simulation reached a quasi-steady state within one ion gyroperiod.)

Before considering the obvious differences between the 1-D and 2-D simulations, we note that in both cases the plasma has a potential that is negative with respect to the wall, by a few times the ion temperature $T_{i}$, with most of the change in potential occuring in a narrow sheath near the wall. Transport across a magnetic field is more rapid for ions than for electrons, so in steady state the plasma must develop a potential that is negative with respect to the wall, to make the ion and electron loss rates equal. This is in contrast to the case where the magnetic field is perpendicular to the wall, which results in a positive potential with respect to the wall. (When the magnetic field is at an oblique angle $\theta$ to the wall, unless $\theta$ is extremely small the flow of electrons to the wall along the magnetic field will be much greater than the transport of ions across the magnetic field, and the plasma will have a positive potential with respect to the wall, of a magnitude that is almost independent of $\theta .5,6$ )

There is another important difference between cross-field sheaths and sheaths developing along a mrgnetic field (or in an unmagnetized plasma). While it is possible to make a collisicniess sheath model along a magnetic field, which may be modified when collisions are included, it is not possible to model a cross-field sheath without collisions (or effective collisions of some kind) to give cross-field transport. The form of the sheath will depend very much on the details of the cross-field transport used in the model. (Daybelge and Bein ${ }^{5}$ calculate the profile of a "collisionless" sheath in the limit $\theta=0$ by assuming that, the ion and electron distribution functions are zero in the rugions of phase space where particles go the wall, and maxwellian everywhere else. But their calculation, which gives results quite different from our simulations, does not describe a self-consistent steady state on the time scale of cross-field transport, and has no relevance to the late time behavior. of our simulation models.) 
The differences seen in the 1-D and 2-D simulations shown in Figures 1 and 2 are due to the differences in cross-field transport. Initially, ions and electrons are uniformly distributed in $x$, the potential $\Phi(x)$ is flat, and those particles whose guiding center position $x_{g c}=x+v_{y} / \Omega$ is within a Larmor radius $v_{\perp} / \Omega$ of the well can be lost immediately, without any transport in $x_{g c}$. (Here $\Omega$ is the gyrofiequency, either $\Omega_{i}=e B_{0} / m_{i} c$, or $\Omega_{e}=-e B_{0} / r \imath_{e} c$, and $v_{\perp}=v_{s}^{2}+v_{y}^{\pi /}$ ) Since an ion Larmor radius is greater than an electron Larmor radius, more ions than electrons can be lost, and the plasma will develop a negative potential. If the ion plasma frequency $\omega_{p i}$ is much greater than $\Omega_{i}$, then the plasma will build up a negative potential $\Phi_{0}$ of a few times $T_{i} / e$ when only a small fraction of the ions within a thermal Larmor radius of the wall have been lost, viz. when those ions within a few Debye lengths of the wall have been lost, and after this the ion losses will almost cease. At this time the plasma is quasineutral with uniform density, and the potential profile $\Phi(x)$ is flat, except in sheaths a few Debye lengths wide (or an electron Larmor radius wide if that is greater than a few Debye lengths) near the walls. All of the rise in potential occurs in these sheaths, which have much lower density than the rest of the plasma. In the 1-D simulation, there are no forces in the $y$-direction exerted on particles, so there can be no change in a particle's $x_{g e}$, which is the $y$-component of its canonical momentum. The potential and density profiles therefore remain in this state, as is seen in Fig. 1. Actually this is not strictly speaking a steady state, since particles can diffuse in energy, as a result of collisions with other particles in which part of the $x$-component of momentum is exchanged. Even a particle with $x_{g e}$ not within a Larmor radius of the wall can be lost, with no change in its $x_{g c}$, if it diffuses up far enough in energy so that its Larmor radius becomes greater than the distance from $x_{g c}$ to the wall. The further $x_{g c}$ is from the wall, the longer this takes. Eventually, the plasma density profile will evolve to a narrow peak equidistant from the two walls, but if the width of the slab $2 R_{p}$ is much greater than a thermal ion Larmor radius $\rho_{i}$ then the plasma will take an exponentially long time to reach this state. For any reasonable number of time steps in the simulation, the plasma density and potential will be essentially flat except in narrow sheaths near the wall, as seen in Fig. 1.

In a 2-D simulation, on the other hand, particles can change their $x_{g c}$, as a result of forces in the $y$-direction, either due to binary collisions with other particles, or due to interractione with waves which have a finite $y$-component of wavenumber, $k_{y}$. If the ions have a characteristic collision time $\nu_{i}$, then the time required for an ion to diffuse 
Figure 1

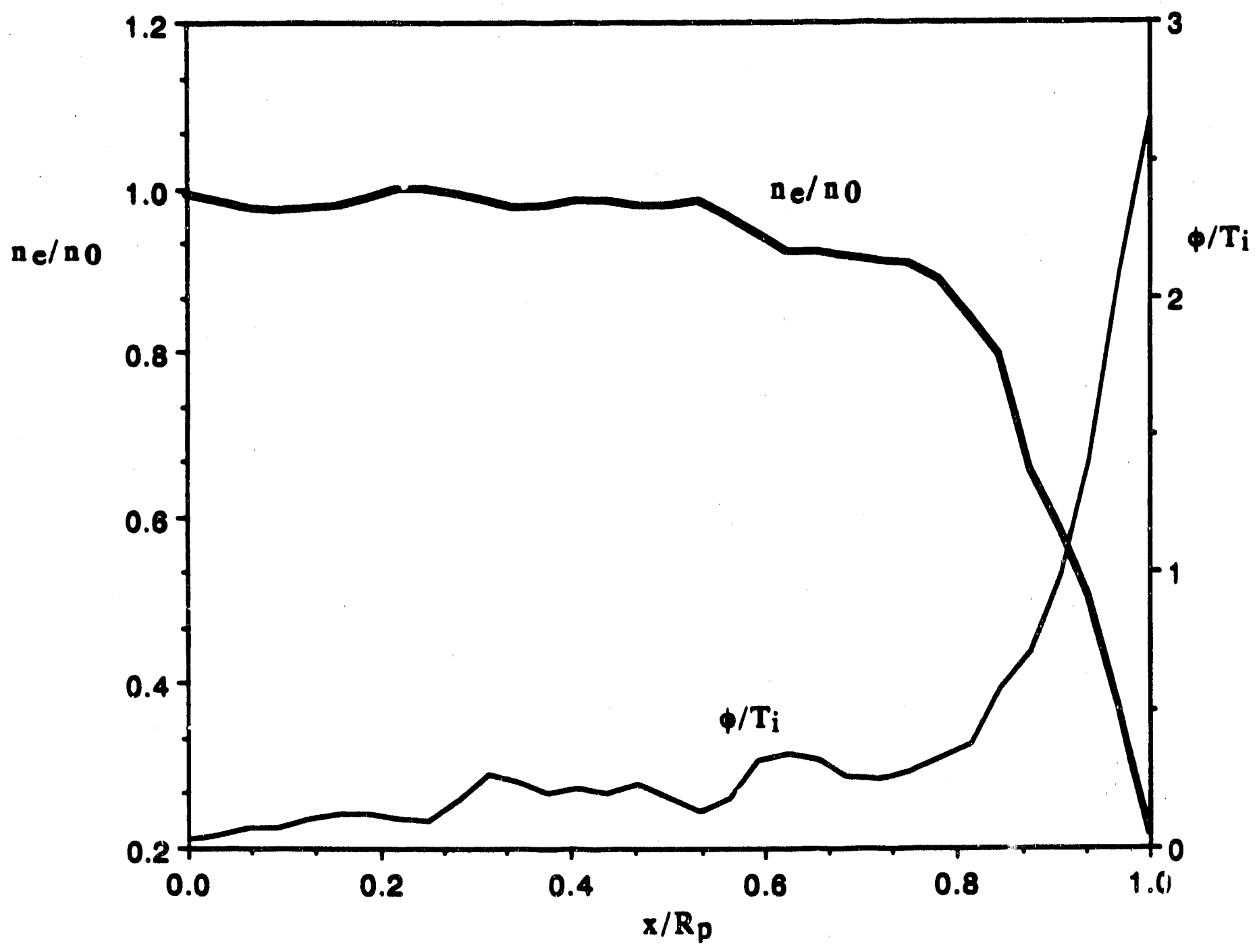

1. Potential and electron density profiles for a 1-D simulation with no diffusion of $\boldsymbol{x}_{g \mathrm{gc}}$. Debye length and thermal electron Larmor radius are both $0.03 R_{p}$, and thermal ion Larmor radius is $0.2 R_{p}$. 
to the wall will be on the order of $R_{p}^{2} / \rho_{i}^{2} \nu_{i}$, and the electrons will diffuse to the wall in a characteristic time $R_{p}^{2} / \rho_{e}^{2} \nu_{e}$. Since the tlow to the wall must be ambipolar, the species with the shorter diffusion time, usually the ions, will be held back by an electric field, and both species will diffuse to the wall in a diffusion time characteristic of the more slowly diffusing species, usually the electrons. After a time longer than this, the density profile in the quasineutral region, away from the sheaths near the walls, will be determined by the balance between diffusion in $x_{g e}$ and the source $S(x)$

$$
D_{2} \frac{d^{2} n_{e}}{d x^{2}}+S(x)=0
$$

where $D_{z} \approx \rho_{e}^{2} \nu_{e}$ is the diffug: roefficient in electron guiding center position. We have neglected the effect of the electric field on the electrons. If $D_{x}$ and $S$ are independent of $x$, then the density profile will be parabolic, with a maximum in the center of the slab, at $x=0$, and going to zero at the walls. The potential profile in the quasineutral region will depend on the ion energy distribution, which in turn will depend on the balance between diffusion and loss of energy, and spatial diffusion, but in general the potential will have a logarithmic dependence on density in this region, which is analogous to the Bohm presheath ${ }^{7}$. Near the walls there will be sheaths about a Debye length wide, with a Debye length defined not by the density in the center of the plasma, but by the much lower density near the wall, at the beginning of the sheath. Hence, for the same central density and temperature, the sheaths in this case will be substantially wider than in the 1-D case. For a parabolic density profile, the sheath width will be approximately

$$
\delta x=\left(R_{p} / 2\right)^{1 / 3} \lambda_{D}^{2 / 3}
$$

where $\lambda_{D}$ is the Debye length in the center of the plasma. Figure 2 shuws that the density and potential profiles of the 2-D simulation are in approximate agreement with this picture, although there are details, such as a macroscopic vortex structure resulting from the nonlinear development of a Kelvin-Helmholtz instability, ${ }^{8}$ that cannot be described by spatial diffusion and an effective collision rate.

The fact that the density profile in the 2-D simulation is described fairly well as a parabola going to zero at the wall, suggests that much of the 2-D physics, which makes the 2-D simulation look very different from the $1-\mathrm{D}$ simulation, could be included in a $1-\mathrm{D}$ simulation by artificially putting in a coefficient of diffusion in $x_{g c}$, uniform in space. This diffusion coefficient would incorporate all of the 2-D processes, such as ion-electron 


\section{Flgure 2}

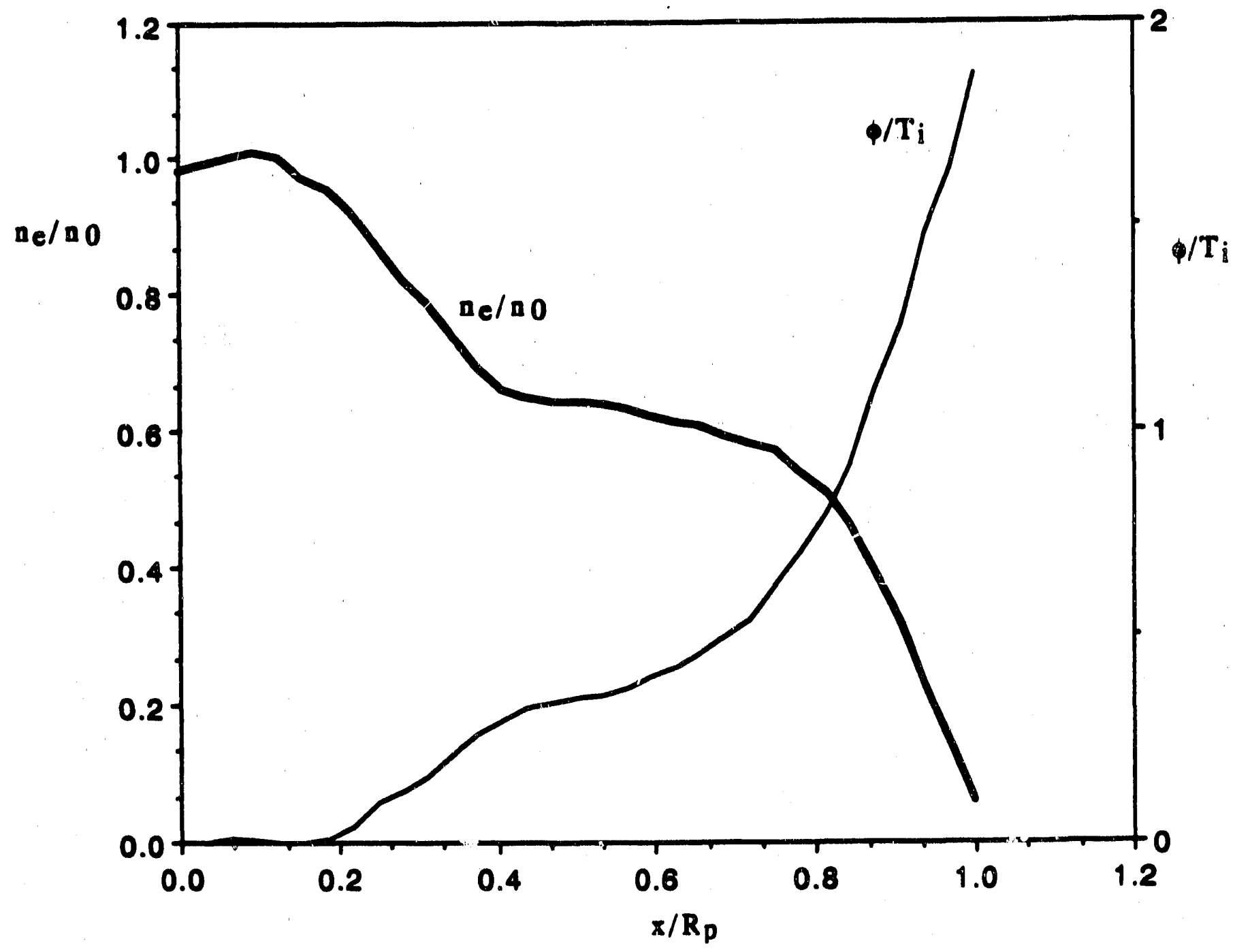

2. Potential and electron density profiles for a 2-D simulation, with central Debye length $0.06 R_{p}$, edge Debye length $0.12 R_{p}$, and thermal ion Larmor radius $0.18 R_{p}$. 
collisions, Kelvin-Helmholtz instabilities, ${ }^{9}$ and lower hybrid drift waves, ${ }^{10}$ which cause diffusion in $x_{g c}$, and would allow all of the bulk properties of the 2-D simulation (although not, of course, such details as the Kelvin-Heimholtz vortex structure) to be reproduced in a much cheaper 1-D simulation. A nontrivial check of such a notion would be to see whether the shape of the potential profile $\Phi(x)$ could also be reproduced, since this profile depends on the interraction between energy diffusion and drag, which already are present in the 1-D simulation, and spatial diffusion.

In Sec. II, we find the potential and density profiles that would be found in the 1-D case, with no diffusion in $x_{g c}$, when the plasma has reached a quasi-steady state, with further changes (due to diffusion in energy) being exponentially slow. These profiles are compared to those seen in. Fig. 1. In Sec. III, we calculate analytically what the density and potential profiles should look like with uniform ion and electron spatial diffusion coefficients, considering first the limit where the ion spatial diffusion rate is much lower than the energy diffusion rate. This limit is the appropriate one for the 2-D simulation whose results are shown in Fig. 2 , at least for $x$ not too close to the wall, since it involves only one species of ions. Because collisions between like particles cannot result in any diffusion in $x_{g e}$, although they can result in diffusion or loss in energy, the ion spatial diffusion rate in this simulation is due only to collisions with electrons, or to interractions with waves, and is consequently much lower than the energy diffusion rate. It is shown that the potential profile in Fig. 2 is in good quantitative agreement both with the analytic expression (a simple Boltzmann relation between ion density and potential), and with the potential profile of a 1-D simulation in which a weak spatial diffusion rate has been artificially included. Near the wall, however, the simulation results are not in such good agreement with the analytic expression for the potential, because the ion spatial diffusion rate is greater than the energy diffusion rate. If there is more than one species of ion present, as there often is at the edge region of tokamaks, due to impurities, then this limit can also apply further from the wall. An analytic expression for the potential profile is derived in this limit, and is shown to agree well with 1-D simulations in which a strong ion spacial diffusion coefficient has been artificially included.

The analysis in Sec. III does not include finite ion Larmor radius. This is appropriate for simulations, such as the 2-D simulation shown in Fig. 2, where the ion Larmor radius is less than or comparable to the Debye length at the beginning of the sheath, and indeed this tends to be marginally true in the scrape-off layers of tokamaks. At higher density, 
such that $\omega_{p i} \gg \Omega_{i}$ at the beginning of the sheath, finite ion Larmor radius must be taken into account, and there are modifications in the potential within an ion Larmor radius of the wall, even when this is much greater than a Debye length; this region, which is quasineutral, has been called the "magnetic presheath" by Chodura. ${ }^{\circ}$ In Sec. IV we calculate the potential and density profiles including the effect of finite ion Larmor radius, and show that, in the limit that the ion spatial diffusion rate is much greater than the energy diffusion and loss rates, finite ion Larmor radius has no effect; the potential profile found in Sec. III in this limit is exactly valid for arbitrary ion Larmor radius. In the case where the ion spatial diffusion rate is less than the energy diffusion and loss rates, there are some modifications to the potential in the magnetic presheath, within an ion Larmor radius of the wall. However, most of the drop in potential still takes place in the much narrower non-neutral Debye sheath, of width comparable to a Debye length, near the wall. (A similar calculation was done by Holland, Fried and Morales, ${ }^{11}$ who also found a Debye sheath and a magnetic presheath, but not a Bohm presheath because their source was located at $x \rightarrow-\infty$ rather than being distributed throughout the plasma.) This is confirmed by 1-D simulations, with spatial diffusion artificially included, and with different values for the ion Larmor radius. An argument is given suggesting that this would not be true in a 2-D simulation, where ion-electron collisions and current-driven instabilities might be expected to broaden the Debye sheath, resulting in a potential profile whose scale length near the wall is not a Debye length, but an ion Larmor radius. We have not been able to do a 2-D simulation to confirm this conjecture, since such a simulation, with $\omega_{p i} \gg \Omega_{i}$, would require many more particles than the simulation shown in Fig. 2, and would be very expensive. A summary and conclusions are given in Sec. V.

\section{1-D Model Without Diffusion}

Suppose the ions and electrons are initially maxwellian and uniformly distributed in $\boldsymbol{x}_{g c}$

$$
\begin{aligned}
& f_{i}\left(\epsilon, x_{g c}\right)=n_{0} m_{i} T_{i}^{-1} \exp \left(-\epsilon / T_{i}\right) \\
& f_{e}\left(\epsilon, x_{g c}\right)=n_{0} m_{e} T_{e}^{-1} \exp \left(-\epsilon / T_{e}\right)
\end{aligned}
$$

where, for the ions

$$
\begin{gathered}
\epsilon=\frac{1}{2} m_{i}\left(v_{x}^{2}+v_{y}^{2}\right)+e \Phi(x) \\
x_{g c}=x+v_{y} / \Omega_{i}
\end{gathered}
$$


and for the electrons

$$
\begin{gathered}
\epsilon=\frac{1}{2} m_{e}\left(v_{z}^{2}+v_{y}^{2}\right)-e \Phi(x) \\
x_{g c}=x-v_{y} / \Omega_{e}
\end{gathered}
$$

Eqs.(1) and (2) may be written as

$$
\begin{aligned}
& f_{i}=n_{0} m_{i} T_{i}^{-1} \exp \left(-e \Phi(x) / T_{i}\right) \exp \left(-m_{i} v_{x}^{2} / 2 T_{i}\right) \exp \left(-m_{i} v_{y}^{2} / 2 T_{i}\right) \\
& f_{e}=n_{0} m_{e} T_{e}^{-1} \exp \left(+e \Phi(x) / T_{e}\right) \exp \left(-m_{i} v_{x}^{2} / 2 T_{e}\right) \exp \left(-m_{i} v_{y}^{2} / 2 T_{e}\right)
\end{aligned}
$$

and the ion and electron densities are

$$
\begin{aligned}
& n_{i}(x)=n_{0} m_{i} T_{i}^{-1} \exp \left(-e \Phi(x) / T_{i}\right) \int_{-\infty}^{+\infty} d v_{x} \exp \left(-m_{i} v_{x}^{2} / 2 T_{i}\right) \int_{-\infty}^{+\infty} d v_{y} \exp \left(-m_{i} v_{y}^{2} / 2 T_{i}\right) \\
& n_{e}(x)=n_{0} m_{e} T_{e}^{-1} \exp \left(+e \Phi(x) / T_{e}\right) \int_{-\infty}^{+\infty} d v_{x} \exp \left(-m_{e} v_{x}^{2} / 2 T_{e}\right) \int_{-\infty}^{+\infty} d v_{y} \exp \left(-m_{e} v_{y}^{2} / 2 T_{e}\right)
\end{aligned}
$$

Poisson's equation

$$
\frac{d^{2}}{d x^{2}} \Phi=4 \pi e\left(n_{e}-n_{i}\right)
$$

determines $\Phi(x)$, which in this case is equal to 0 , initially. Within a gyroperiod, particles with $\epsilon$ and $x_{g c}$ such that $v_{v}>0$ at $x= \pm R_{p}$ will go to the walls and be lost. The condition for this is

$$
\epsilon>\frac{1}{2} m_{i}\left(x_{g c}-R_{p}\right)^{2} \Omega_{i}^{2}+e \Phi\left(R_{p}\right)
$$

for ions, and

$$
\epsilon>\frac{1}{2} m_{e}\left(x_{g c}-R_{p}\right)^{2} \Omega_{e}^{2}-e \Phi\left(R_{p}\right)
$$

for electrons. At this time, before any diffusion in $\epsilon$ has taken place, the distribution functions will just ie maxwellians truncated in this region. $\Phi(x)$ will no longer be equal to zero everywhere, but will adjust so that Poisson's equation is satisfied with these new distribution functions. Changing variables from $v_{x}$ and $v_{y}$ to $\psi=m_{i} v_{x}^{2} / 2 T_{i}$ and $\xi=$ $\left(m_{i} v_{y}^{2} / 2 T_{i}\right)^{1 / 2}$, the new ion density is

$$
n_{i}(x)=\pi^{-1} n_{0} T_{i}^{-1} \exp \left(-e \Phi / T_{i}\right) \int_{-\infty}^{\xi_{\text {max }}} d \xi \exp \left(-\xi^{2}\right) \int_{0}^{\psi_{\max }} d \psi \psi^{-1 / 2} e^{-\psi}
$$

where $\psi_{\max }$ is the $\psi$ at which an ion can get to the wall, from Eq.(9)

$$
\psi_{\max }=\left(\frac{1}{2} m_{i}\left[\left(x_{g c}-R_{p}\right)^{2}-\left(x_{g c}-x\right)^{2}\right] \Omega_{i}^{2}+e\left[\Phi\left(R_{p}\right)-\Phi(x)\right]\right) / T_{i}
$$


or, expressed in terms of $\xi$

$$
\psi_{\max }=\left(2 m_{i} / T_{i}\right)^{1 / 2} \Omega_{i}\left(\xi_{\max }-\xi\right)\left(R_{p}-x\right)
$$

and $\xi_{\max }$ is the $\xi$ at which $\psi_{\max }=0$

$$
\xi_{\text {max }}=\left(\frac{m_{i}}{8 T_{i}}\right)^{1 / 2}\left(R_{p}-x\right) \Omega_{i}+\frac{e\left[\Phi\left(R_{p}\right)-\Phi(x)\right]}{\left(2 m_{i} T_{i}\right)^{1 / 2}\left(R_{p}-x\right) \Omega_{i}}
$$

Similar expressions hold for the electjons, but with $e$ replaced by $-e$ in the second term of the expression for $\xi_{\text {max }}$, and with $\Omega_{i}, T_{i}$ and $m_{i}$ replaced by $\Omega_{e}, T_{e}$ and $m_{i}$. The $\Omega_{i}$ appearing in Eqs.(13) and (14) is really $\left|\Omega_{i}\right|$, so does not change sign when replaced by $\Omega_{e}$ in the electron expressions.

The $\psi$ integral in Eq.(11) is

$$
\int_{0}^{\psi_{\max }} d \psi \psi^{-1 / 2} e^{-\psi}=\operatorname{erf}\left(\sqrt{\psi_{\max }}\right)
$$

Assuming, as will be shown a posteriori, that the potential drops by more than $T_{i} / e$ from the wall potential in a distance much less than a thermal ion Larmor radius from the wall, we find that $\psi_{\max } \gg 1$ (in which case $\operatorname{erf}\left(\sqrt{\psi_{\max }}\right) \approx 1$ ) unless $R_{p}-x \ll\left(T_{i} / m_{i}\right)^{1 / 2} / \Omega_{i}$, in which case $\psi_{\text {max }} \approx e\left[\Phi\left(R_{p}\right)-\Phi(x)\right] / T_{i}$. In either case

$$
\operatorname{erf}\left(\sqrt{\psi_{\max }}\right) \approx \operatorname{erf}\left[\left(\frac{e\left[\Phi\left(R_{p}\right)-\Phi(x)\right]}{T_{i}}\right)^{1 / 2}\right]
$$

independent of $\xi_{\max }$. Then, from Eqs.(11) and (15)

$$
n_{i}=n_{0} \exp \left(\frac{-e \Phi(x)}{T_{i}}\right) \operatorname{erf}\left(\xi_{\text {max }}\right) \operatorname{erf}\left[\left(\frac{e\left[\Phi\left(R_{p}\right)-\Phi(x)\right]}{T_{i}}\right)^{1 / 2}\right]
$$

The assumption that the potential drops more than $T_{i} / e$ in much less than an ion Larmor radius from the wall also implies that $\xi_{\max } \gg 1$ for any $x$. Then, from Eq.(16),

$$
n_{i}(x)=n_{0} \exp \left(\frac{-e \Phi(x)}{T_{i}}\right) \operatorname{erf}\left[\left(\frac{e\left[\Phi\left(R_{p}\right)-\Phi(x)\right]}{T_{i}}\right)^{1 / 2}\right]
$$

This expression applies at a time on the order of an ion gyroperiod after the particles are initially loaded with uniform density, but before any diffusion in energy has taken place, so the ion energy distribution cuts of sharply at the maximum energy given by Eq.(12). After 
a few energy diffusion times, this sharp cutoff in the distribution function will be smoothed out, and the distribution function will go linearly to zero at the maximum energy, rather than going to zero discontinuously. This situation may be modelled by using

$$
\begin{aligned}
n_{i}(x)=n_{0} \exp \left(\frac{-e \Phi(x)}{T_{i}}\right) \operatorname{erf} & {\left[\left(\frac{e\left[\Phi\left(R_{p}\right)-\Phi(x)\right]}{T_{i}}\right)^{1 / 2}\right] } \\
& -n_{0} \exp \left(\frac{-e \Phi\left(R_{p}\right)}{T_{i}}\right)\left(\frac{e\left[\Phi\left(R_{p}\right)-\Phi(x)\right]}{T_{i}}\right)^{1 / 2}
\end{aligned}
$$

instead of Eq.(17). This will have only a minor effect on the potentia! profile we will calculate, so for simplicity we will uo the original expression for $n_{i}(x)$, Eq.(17).

For the electrons, when $x$ is more than a few electron Larmor radii from the wall, then $\psi_{\text {mas }} \gg 1$ and $\xi_{\text {mas }} \gg 1$, so

$$
n_{e}(x) \approx n_{0} \exp \left(\frac{+e \Phi(x)}{T_{e}}\right)
$$

but within a few Larmor radii of the wall $\psi_{\text {max }}$ is in general neither large nor independent of $\xi_{\text {inas }}$, and we must use

$n_{e}(x)=n_{0} \exp \left(\frac{+e \Phi(x)}{T_{e}}\right) \int_{-\infty}^{\xi_{\text {max }}} d \xi \exp \left(-\xi^{2}\right) \operatorname{erf}\left[\left(2 m_{e} / T_{e}\right)^{1 / 4} \Omega_{e}^{1 / 2}\left(R_{e}-x\right)^{1 / 2}\left(\xi_{\text {max }}-\xi\right)^{1 / 2}\right]$

where

$$
\xi_{\text {mex }}=\left(\frac{m_{e}}{8 T_{e}}\right)^{1 / 2}\left(R_{p}-x\right) \Omega_{e}-\frac{e\left[\Phi\left(R_{p}\right)-\Phi(x)\right]}{\left(2 m_{e} T_{e}\right)^{1 / 2}\left(R_{p}-x\right)}
$$

The potential $\Phi(x)$ may now be found by putting Eqs.(17) and (20) for $n_{i}(x)$ and $n_{e}(x)$ into Poisson's equation. We will not solve for $\Phi(x)$ exactly, but will comment on its qualitative behavior. In the case where the Debye length is much greater than an electron Larmor radius, Eq.(19) may be used for the electrons whcis $R_{p}-x$ is greater than or comparable to a Debye length, and Poisson's equation becornes

$$
\frac{d^{2} \Phi}{d x^{2}}=4 \pi \operatorname{ert} 0\left\{\exp \left(\frac{+e \Phi(x)}{T_{e}}\right)-\exp \left(\frac{-e \Phi(x)}{T_{i}}\right) \operatorname{erf}\left[\left(\frac{e\left[\Phi\left(R_{p}\right)-\Phi(x)\right]}{T_{i}}\right)^{1 / 2}\right]\right\}
$$

In this case the potential drops from $\Phi\left(R_{p}\right)$ down to $\Phi_{0}$, which is a drop of a few times $T_{i} / e_{4}$ in a sheath about a Debye length thick, and is close to $\Phi_{0}$ in the rest of the plasma. Nothing special happens when $R_{p}-x$ is comparable to an ion Larmor radius. When 
$R_{p}-x$ is comparable to an election Larmor radius, we must use Eq.(20) for $n_{e}$, and $\xi_{\max }$ is positive, approaching 0 as $x$ approaches the wall. The electron density is reduced by a factor of 2 in this region, but over this short distance the reduction in electron density has little effect on the potential. In the opposite limit, where the Debye length is much less than an electron Larmor radius, Eq.(20) must be used for the electron density throughout the sheath, and $\xi$ would be very negative if the potential dropped more than $T_{e} / e$ in a distance much less than an electron Larmor radius. In this case $n_{e}$ from Eq.(20) would be exponentially small throughout the sheath, and Poisson's equation could not he satisfied. Fience the potential canonot drop by $T_{e} / e$ over a distance much less than an electron Larmor radius from the wall. This will make $\xi_{\text {mas }} \approx-1$ in the sheath, which will be about an electron Larmor radius wide, and allow Poisson's equation to be satisfied.

In summary, in the case of a 1-D simu'ation with no diffisi: in $x_{g c}$ we expect that the potential will be nearly flat in most of the plasma, ana will rise up to the wall potential (a few $T_{i} / e$ ) in a sheath of width comparable to the greater of the Debye length and the electron Larmor radius. This is exactly what is seen in the 1-D simulation result shown in Fig. 1. A series of 1-D simulations with different values of the Debye length ${ }^{12}$ shows that the sheath width scales with the Debye length.

\section{1-D Model With Diffusion}

The ion and electron distribution functions are $f_{i}\left(\epsilon, x_{g c}\right)$ and $f_{e}\left(\epsilon, x_{g c}\right)$, where $\epsilon$ is the energy and $x_{g c}$ is the guiding center position. In steady state $d f_{e} / d t=0$ and $d f_{i} / d t=0$, so

$$
\begin{aligned}
& \frac{\partial}{\partial x_{g c}} D_{z, i} \frac{\partial}{\partial x_{g c}} f_{i}+\frac{\partial}{\partial x_{g c}} V_{i} f_{i}+\frac{\partial}{\partial \epsilon} D_{\epsilon, i} \frac{\partial}{\partial \epsilon} f_{i}+\frac{\partial}{\partial \epsilon} G_{i} f_{i}+S_{i}=0 \\
& \frac{\partial}{\partial x_{g c}} D_{z, e} \frac{\partial}{\partial x_{g c}} f_{e}+\frac{\partial}{\partial x_{g c}} V_{e} f_{e}+\frac{\partial}{\partial \epsilon} D_{e, e} \frac{\partial}{\partial \epsilon} f_{e}+\frac{\partial}{\partial \epsilon} G_{e} f_{e}+S_{e}=0
\end{aligned}
$$

where (for each specier) $D_{x}$ is the spatial diffusion coefficient, $V$ is the radial flow velocity due to a force in the $y$-direction (which may occur, as a resuli of collisions or instabilities, in 2-D, and could also be imposed artificially in 1-D), $D_{\epsilon}$ is the diffusion zoefficient in energy, $G$ is the energy loss rate, and $S$ is the source. The coefficient: $D_{x}, V, D_{\varepsilon}, G$, and $S$ are all, in general, functions of $\epsilon$ and $x_{g c}$. If the sources are locally maxwellian and i when integrated over energy) independent of $x$, then $S_{e}$ will be proportional to $\exp \left[(-\epsilon-e \Phi(x)) / T_{e}\right]$ for $\epsilon>--e \Phi(x)$ and zero otherwise, while $S_{i}$ will be proportional to $\exp \left[(-\epsilon+e \Phi(x)) / T_{i}\right]$ for

$\epsilon>\boldsymbol{e} \Phi(x)$, and zero otherwise. (Although $x$ has a complicated dependence on $x_{g c}$ and $\epsilon$ near the walls, where $\Phi(x)$ has a scale length less than or comparable to a La.mor radius, 
the source terms are not important in these regions, whereas far from the walls $x \approx x_{g c}$.) We have set the radial drift terms $V_{i}$ and $V_{e}$ equal to zero in our 1-D simulations, since we have found that it is not needed to reproduce the results of the 2-D simulations, although, for reasons given in Sec. V, such a term may be needed to reproduce 2-D simulations at higher density.

We first consider the case where the energy diffusion and energy loss rate for each spicies is inuch greater than the diffusion rate in $x_{g c}$ for that species. This is appropriate for the 2-D simulation shown in Fig. 2, since there is only one species of ions, and there is no contribution to ion spatial diffusion from ion-ion collisions, only from ion-electron collisions (which have a collision rate that is lower than the ion-ion collision rate by the mass ratio) and perhepis from interractions of ions with waves. Ion-ion collisions can, however, cause energy diffusion. The dominant terms of Eqs.(23) and (2.3) are then

$$
\begin{aligned}
& \frac{\partial}{\partial \epsilon} D_{\epsilon, i} \frac{\partial}{\partial \epsilon} f_{i}+\frac{\partial}{\partial \epsilon} G_{i} f_{i}=0 \\
& \frac{\partial}{\partial \epsilon} D_{\epsilon, e} \frac{\partial}{\partial \epsilon} f_{e}+\frac{\partial}{\partial \epsilon} G_{e} f_{\epsilon}=0
\end{aligned}
$$

subject to the boundary conditions $f_{i, e}\left(\epsilon, x_{g c}\right) \rightarrow 0$ as $\epsilon \rightarrow \infty$. The source terms have been neglected, since, in steady state, they must be equal to the losses, which are due to spatial diffusion and flow, which have been assumed to be small compared to the energy diffusion and energy loss terms. The energy diffusion and energy loss terms for the electrons are dominated by Coulomb collisions with electrons, and the energy diffusion term for the ions is dominated by Coulomb collisions with ions. Unless $T_{e} \ll T_{i}$, the energy loss term for ions is also dominated by Coulomb collisions with ions. If we neglect finite Larmor radius, so that $x_{g c} \approx x$, then the energy diffusion and energy loss terms at a given $x_{g c}$ depend only on the distribution function at that $x_{g c}$. Eqs.(25) and (26) then imply ${ }^{13}$ that the ion and electron distribution functions at a given $x_{g c}$ are maxwellian

$$
\begin{aligned}
f_{i}\left(\epsilon, x_{g c}\right) & =\frac{n_{i}\left(x_{g c}\right)}{T_{i}\left(x_{g c}\right)} \exp \left(\frac{-\epsilon+e \Phi\left(x_{g c}\right)}{T_{i}\left(x_{g c}\right)}\right) \text { for } \epsilon \geq e \Phi\left(x_{g c}\right) \\
& =0 \text { for } \epsilon<e \Phi\left(x_{g c}\right) \\
f_{e}\left(\epsilon, x_{g c}\right) & =\frac{i_{e}\left(x_{g c}\right)}{T_{e}\left(x_{g c}\right)} \exp \left(\frac{-\epsilon-e \Phi\left(x_{g c}\right)}{T_{e}\left(x_{g c}\right)}\right) \text { for } \epsilon \geq-e \Phi\left(x_{g c}\right) \\
& =0 \text { fol: } \epsilon<-e \Phi\left(x_{g c}\right)
\end{aligned}
$$


where we have used the approximation $\Phi(x) \approx \Phi\left(x_{g c}\right)$. The densities and temperatures are determined by the spatial diffusion and source terms in Eqs.(23) and (24) which were neglected in Eqs.(25) and (26). If we integrate Eqs.(23) and (24) over c, then the dominant terms, the energy diffusion and energy loss terms, vanish, because the Coulomb collison operator conserves particles. This yields one equation for $n_{i}\left(x_{g c}\right)$ and $T_{i}\left(x_{g c}\right)$ and one equation for $n_{e}\left(x_{g c}\right)$ and $T_{e}\left(x_{g c}\right)$. The source term may be neglected for the ions, since it is equal to the electron source term, hence comparable to the electron spatial diffusion term and much less than the ion spatial diffusion term. The remaining ion term, the spatial diffusion term, may then be integrated over $x_{g e}$, giving

$$
\begin{aligned}
\left(\int_{e \Phi}^{\infty} d \epsilon D_{z, i} e^{-(e-e \xi) / T_{i}}\right)\left(\frac{d n_{i}}{d x_{g c}}+\frac{e n_{i}}{T_{i}} \frac{d \Phi}{d x_{g c}}\right)+ \\
\left(\int_{e \Phi}^{\infty} d \epsilon\left(\epsilon-e \Phi-T_{i}\right) D_{z, i} e^{-(e-e \Phi) / T_{i}}\right) \frac{n_{i}}{T_{i}^{2}} \frac{d T_{i}}{d x_{g c}}=\text { const }
\end{aligned}
$$

The constant on the right hand side of Eq.(29) is equal to zero if the plasma slab is symmetric about $x=0$, i.e. if $\Phi\left(R_{p}\right)=\Phi\left(-R_{p}\right)$ and the source terms are symmetric about $x$. The electron equation is

$$
\begin{aligned}
& \frac{d}{d x_{g c}}\left(\int_{-e \Phi}^{\infty} d \epsilon D_{x, e} e^{-(\epsilon+e \Phi) / T_{e}}\right)\left(\frac{d n_{e}}{d x_{g c}}-\frac{e n_{e}}{T_{e}} \frac{d \Phi}{d x_{g c}}\right)+ \\
& \frac{d}{d x_{g c}}\left(\int_{-e \Phi}^{\infty} d \epsilon\left(\epsilon+e \Phi-T_{e}\right) D_{z, e} e^{-(\epsilon+e \Phi) / T_{e}}\right) \frac{n_{e}}{T_{e}^{2}} \frac{d T_{e}}{d x_{g c}}+\int_{-e \Phi}^{\infty} d \epsilon S_{e} e^{-(e+e \Phi) / T_{e}}=0
\end{aligned}
$$

Another pair of equations for the ion and electron temperatures and densitics may be obtained by multiplying Eqs.(23) and (24) by $\epsilon$ and then integrating over $\epsilon$. This again causes the ciominant energy diffusion and energy loss terms to vanish, since the Coulomb collision operator conserves energy, and we neglect transfer of energy between ions and electrons, which we assume takes place on a time scale even longer than the electron spatial diffusion time. We integrate the resulting ion equation over $x_{g c}$, and subtract $e \Phi$ times the left hand side of Eq.(29), to obtain

$$
\begin{aligned}
\left(\int_{e \Phi}^{\infty} d \epsilon(\epsilon-e \Phi) D_{x, i} e^{-(\epsilon-e \Phi) / T_{i}}\right)\left(\frac{1}{T_{i}} \frac{d n_{i}}{d x_{g c}}+\frac{e n_{i}}{T_{i}^{2}} \frac{d \Phi}{d x_{g c}}\right)+ \\
\left(\int_{e \Phi}^{\infty} d \epsilon(\epsilon-e \Phi)\left(\epsilon-e \Phi-T_{i}\right) D_{x, i} e^{-(\epsilon-e \Phi) / T_{i}}\right) \frac{n_{i}}{T_{i}^{3}} \frac{d T_{i}}{d x_{g c}}=0
\end{aligned}
$$

while the electron equation is

$$
\frac{d}{d x_{g c}}\left(\int_{-e \Phi}^{\infty} d \epsilon(\epsilon+e \Phi) D_{\varepsilon, e} e^{-(\epsilon+e \Phi) / T_{\bullet}}\right)\left(\frac{1}{T_{e}} \frac{d n_{e}}{d x_{g c}}-\frac{e n_{e}}{T_{e}^{2}} \frac{d \Phi}{d x_{g c}}\right)+
$$




$$
\begin{aligned}
& \frac{d}{d x_{g e}}\left(\int_{-e \Phi}^{\infty} d \epsilon\left(\epsilon+e \Phi-T_{e}\right)(\epsilon+e \Phi) D_{z, e} e^{-(\epsilon+e \Phi) / T_{e}}\right) \frac{n_{e}}{T_{e}^{3}} \frac{d T_{e}}{d x_{g c}}+ \\
& \frac{1}{T_{e}} \int_{-e \Phi}^{\infty} d \epsilon(\epsilon-e \Phi) S_{e} e^{-(e+e \Phi) / T_{e}}=0
\end{aligned}
$$

Note that the two equations for the ions, Eqs.(29) and (31), may be written as

$$
\begin{aligned}
& A_{11}\left(\frac{d n_{i}}{d x_{g c}}+\frac{e n_{i}}{T_{i}} \frac{d \Phi}{d x_{g c}}\right)+A_{12} \frac{d T_{i}}{d x_{g c}}=0 \\
& A_{21}\left(\frac{d n_{i}}{d x_{g c}}+\frac{e n_{i}}{T_{i}} \frac{d \Phi}{d x_{g c}}\right)+A_{22} \frac{d T_{i}}{d x_{g c}}=0
\end{aligned}
$$

where the $A_{i j}$ 's are various energy moments of the diffusion coefficient $D_{s, i}$. Then, unless $A_{11} A_{22}-A_{12} A_{21}$ happens to vanish (and there is no reason why it should),

$$
\frac{d n_{i}}{d x_{g c}}+\frac{e n_{i}}{T_{i}} \frac{d \Phi}{d x_{g c}}=0
$$

and

$$
\frac{d T_{i}}{d x_{g c}}=0
$$

In other words, the ion temperature is the same everywhere, and the ion density is related to the potential by a Boltzmann relation. This simple result does not depend on the details of the collision operator, but only on the fact that $D_{x, i} \gg D_{x, e}$, so that the ion source term $S_{i}$, which is equal to the electron source term $S_{e}$, may be neglected compared to the ion diffusion term. Physically, the reason for this result is that the potential adjusts itself to make the ion particle flux nearly vanish (so that it can be equal to the electron particle flux), by making the $d \Phi / d x$ contribution to the ion particle flux nearly cancel the $d n_{i} / d x$ and $d T_{i} / d x$ contributions. This makes the convective part of the ion heat flux nearly vanish, but there is also a conductive part of the ion heat flux, proportional to $d T_{i} / d x$, which in general will be comparable to one of the terms in the convective part, hence much greater than the convective part. Since there are no sources or sinks of heat for the ions, other than the sources and sinks of particles, the conductive heat flux will rapidly make the ions isothermal, wi'h a temperature $T_{i}$ equal to the mean kinetic energy of the ion source.

No such simple result can be obtained for the electrons, since the electron source term is comparable to the other terms. However, a major simplification of the electron equations may be obtained if we assume that $T_{e}$ is much greater than $e \Phi$. Since, as follows from the 
ion Boltzmann relation, Eq.(34), $e \Phi$ will be comparable to $T_{i}$, this approximation will hold if the mean energy of the electron source is much greater than the mean energy of the ion source. Then the $d \Phi / d x$ term in Eq.(30) may be neglected and Eq.(30) may be written

$$
\frac{d}{d x_{g c}} \bar{D}_{x, e} \frac{d}{d x_{g c}} n_{e}+\bar{S}_{e}=0
$$

where $\bar{D}_{x, e}$ and $\bar{S}_{e}$ are averages over energy. In general $\bar{D}_{x, e}$ will depend on the electron density $n_{e}$ and temperature $T_{e}$, which must be found self-consistently from Eq.(32), which represents a balance between the electron source, convective and conductive heat flow, and sink at the walls. $\bar{D}_{z, e}$ may also depend on the ion density and temperature, and perhaps on turbulence as well. If we make the crude assumption that $z, e$ is independent of $x_{g c}$, take $\bar{S}_{e}$ independent of $x_{g c}$ (as was done in the simulations) and use the bouridary conditions $n_{e}=0$ at $x= \pm R_{p}$, then Eq.(36) gives a parabolic electron density profile. This was true to a good approximation for the 2-D simulation, which suggests that the assumption of uniform diffusion coefficient $\bar{D}_{x, e}$ is not a bad one. This is consistent with the evidence, reported previously, ${ }^{8}$ that the electron diffusion in the simulation is not due primarily to Coulomb collisions, but is dominated by turbulent convective cells, which might be expected to diffuse all electrons at about the same rate.

The ion density and potential may be related to the electron density, which is now assumed to be fixed, by Poisson's equation. For $x$ more than a Debye length from the wall, this means that the plasma is quasineutral, $n_{i} \approx n_{e}$. The potential $\Phi(x)$ in this region, the Bohm presheath, may then be found from the ion Boltzmann relation, Eq.(34). We find

$$
\Phi(x)=\Phi(0)-T_{i} \ln \left(1-x^{2} / R_{p}^{2}\right)
$$

The potential does not really become infinite at $x= \pm R_{p}$, of course, since the density does not quite go to zero at the wall, but is a low value such that the ion flux to the wall is equal to the electron flux to the wall, and the potential at the wall is generally a few times $T_{i} / e$. (An accurate expression for the wall potential cannot be found using Eos.(29) and (30) as a starting point, even if the ion source term is included, since, as will be discussed below, the ion spatial diffusion rate is not small compared to the energy diffusion and energy loss rates near the wall, and the Boltzmann relation for the ions, Eq.(34), is not valid there.)

Figure 3 shows the potential $\Phi(x)$ for a 1-D particle simulation using the PDW1 code, ${ }^{14}$ with spatial diffusion artificially added by including random scattering from fixed scattering centers, in Monte Carlo fashion, by both ions and electrons. This Monte Carlo 
Figure 3

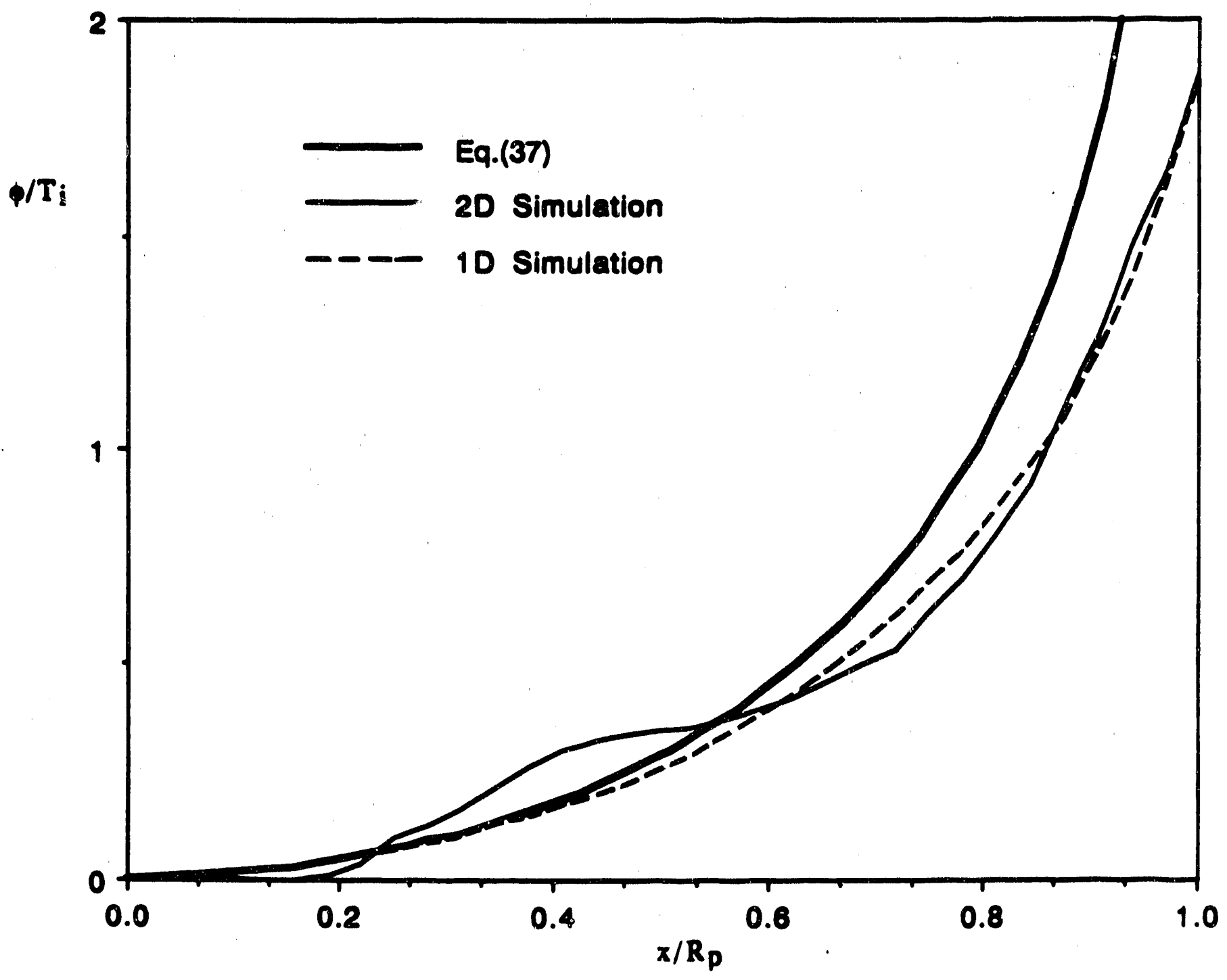

3. Potential profiles from a 2-D simulation, from a 1-D simulation with Monte Carlo diffusion of $x_{g c}$, with similar Debye length and ion Larmor radius, and from Eq.(37). 
collision frequency was made small enough so that the spatial diffusion rate was less than the energy diffusion and energy loss rates (which were due to Coulomb collisions with other charged particles in the simulation). The simulation has been allowed to run for 10,000 time steps, corresponding to 250 ion cyclotron periods, 8 ion collision times, and 1000 electron collision times. An implicit electron mover was used, so that the time step could be set equal to an electron cyclotron period; jn effect only the electron guiding center positions were followed. During the run, about half of the particles went to the wall and were replaced by source particles. The potential from this 1-D simulation is compared to the theoretical $\Phi(x)$ from Eq. (37), and to $\Phi(x)$ for the 2-D simulation shown in Fig. 2. The potential profile from the 1-D simulation is in good agreement with the 2-D simulation, the main difference being a feature in the potential profile of the 2-D simulation caused by a Kelvin-Helmholtz vortex, which of course we would not expect to reproduce in tne 1-D simulation. The potential profile from Eq.(37) differs from that of the 1-D simulation by less than $15 \%$ throughout most of the plasma, but the two become significantly different as $x$ gets close to the wall. This difference is not due to the breakdown of quasineutrality within a Debye length of the wall, since quasineutrality is still a very good approximation in the region where Eq.(37) and the 1-D simulation start to differ, but to the failure of the ordering that the ion diffusion time to the wall, $\left(x-R_{p}\right)^{2} / D_{x, i}$, is much greater than the energy diffusion and energy loss times. This means that the ion distribution function is no longer locally maxwellian, but is reduced at energies above $e\left(\Phi\left(R_{p}\right)-\Phi(x)\right)$, the energy at which an ion can reach the wall and be lost. Then the ion density will fall more rapidly with increasing $\Phi$ than it would if the Boltzmann relation, Eq.(34), were satisfied, and $\Phi(x)$ will not be as high as one would expect from Eq.(37). In this region, $\Phi(x)$ may be found by solving Eqs.(23) and (24) in the opposite limit, where the energy diffusion and energy loss rate for each species is much less than the $x_{g c}$ diffusion rate for that species.

We now consider that limit, taking it as true throughout the slab. (However, we will still assume the ion energy diffusion rate is greater than the electron spatial diffusion rate, otherwise the potential $\Phi(x)$ will be nearly flat, varying much less than $T_{i}$ across the plasma, to avoid having the confinernent time of the coldest ions be greater than that of the electrons.) This is also an appropriate limit for the edge region of a tokamak with a moderately large impurity density. Spatial diffusion for hydrogen ions will be dominated by collisions with impurity ions, but such collisions will not cause much energy diffusion if the impurity ions are much more massive than the hydrogen. As before, we take $V_{i}=V_{e}=0$ 
in Eqs.(23) and (24), and we assume that $T_{e} \gg T_{i}$, which allows us to neglect the effect of the potential (which will have variations across the plasma of order $T_{i}$ ) on the electron flux. The electron distribution is thes given by

$$
D_{x, e} \frac{\partial^{2}}{\partial x^{2}} f_{e}(\epsilon, x)+S_{e}(\epsilon)=0
$$

If $S_{e}(\epsilon)$ goes like $\exp \left(-\epsilon / T_{e}\right)$, and $D_{x, e}$ is uniform and independent of $\epsilon$, then $f_{e}$ has the same dependence on $\epsilon$. Integrating Eq.(38) over $\epsilon$ then gives the electron density profile,

$$
n_{e}(x)=\left(1-\frac{x^{2}}{R_{p}^{2}}\right) n_{0}
$$

where $n_{0}=R_{p}^{2} S / 2 D_{x, e}, S$ is the source $S_{\varepsilon}(\epsilon)$ integrated over $\epsilon$, and we have used the boundary conditions $n_{e}(x)=0$ at the walls, $x= \pm R_{p}$. Equation(39) is valid for $x$ more than a few electron Larmor radii from $\boldsymbol{\Sigma}_{p}$, where $x_{g c} \approx x$ is a good approximation.

To find the potential $\Phi(x)$, the ion diffusion equation is used to find $n_{i}(x)$ in terms of $\Phi(x)$, and then Poisson's equation

$$
\frac{d^{2}}{d x^{2}} \Phi=4 \pi e\left(n_{e}-n_{i}\right)
$$

and Eq.(39) for $n_{e}(x)$ are used to solve simultaneously for $\Phi(x)$ and $n_{i}(x)$. This procedure is considerably simplified if $x$ is more than a few Debye lengths from the wall. Then Poisson's equation implies $n_{i}(x) \approx n_{e}(x)$.

Since we are assuming that the ion energy diffusion rate $D_{\epsilon, i} T_{i}^{-2}$ and energy loss rate $G_{i} T_{i}^{-1}$ are much less than the radial diffusion rate $D_{x, i} R_{p}^{-2}$, and since $D_{x, i} \gg D_{x, e}$, ions with energy greater than the confining potential $e \Phi\left(R_{p}\right)$ will be lost in a time short compared to the average ion loss time (which in steady state must be equal to the average electron loss time), hence $f_{i}\left(\epsilon, x_{g c}\right) \approx 0$ for $\epsilon \geq e \Phi\left(R_{p}\right)$. Ions with energy less than $\epsilon_{\max } \equiv e \Phi\left(R_{p}\right)$ will never be lost until they diffuse in energy up to $\epsilon_{\max }$, at which time they will be lost almost immediately. The ion loss flux will then be equal to

$$
D_{\varepsilon, i} \frac{\partial}{\partial \epsilon} f_{i}-G_{i} f_{i}
$$

evaluated at $\epsilon=\epsilon_{\max }$ and integrated over $x$. 'This loss flux must be equal to the electron loss flux and to the source $S$ integrated over $x$. If, as is reasonable, $G_{i}<D_{\epsilon, i} T_{i}^{-1}$ and $D_{\epsilon, i} T_{i}^{-2} \gg D_{x, e} R_{p}^{-2}$, then $\epsilon_{\max }$ will have to be a few times $T_{i}$, so that the ions are lost 
when they are far out on the tail of the distribution $f_{i}\left(\epsilon, x_{i}\right)$, which will be roughly maxwellian at energies well below $\epsilon_{\operatorname{mas}}$. For ions with $\epsilon<\epsilon_{\max }$, which are almost all of the ions, the radial diffusion term will be much greater than the other terms, which may be neglected. Then Eq.(23) becomes

$$
\frac{\partial}{\partial x_{g c}} D_{x, i} \frac{\partial}{\partial x_{g c}} f_{i}\left(\epsilon, x_{g c}\right)=0
$$

Equation (40) may be integrated over $x_{g c}$, using the fact that (by symmetry) $\partial f_{i} / \partial x_{g c} \approx 0$ at $x_{g c}=0$ (where we may take $\left.\approx x_{g c}\right)$. Then

$$
D_{x, i} \frac{\partial}{\partial x_{g c}} f_{i}\left(\epsilon, x_{g=}\right)=0
$$

so

$$
f_{i}\left(\epsilon, x_{g c}\right)=F(\epsilon)
$$

independent of $x_{g c}$. To find $F(\epsilon)$ it would be necessary to solve a differential equation which includes the energy diffusion, energy loss, and source terms, integrated over the range of $x_{g c}$ for which $e \Phi\left(x_{g c}\right)<\epsilon$ (since radial diffusion takes place on a much faster time scale than the energy diffusion and energy loss), subject to the boundary condition $F\left(\epsilon_{\max }\right)=0$. Since the source term at least will depend on $\Phi\left(x_{g c}\right)$, which is not known yet, and depending on the details of the model the energy diffusion and loss terms may depend on $F(\epsilon)$, it could be a difficult numerical problem to find $F(\epsilon)$. Instead, we will assume that

$$
\begin{aligned}
F(\epsilon) & =\exp \left(-\epsilon / T_{i}\right)-\exp \left(-\epsilon_{\max } / T_{i}\right) \text { for } \epsilon<\epsilon_{\max } \\
& =0 \text { for } \epsilon \geq \epsilon_{\max }
\end{aligned}
$$

which is qualitatively reasonable, and is probably exactly right for some model of energy diffusion and loss. The ion guiding nenter density $n_{i}\left(x_{g c}\right)$ may then be found by integrating $F(\epsilon)$ from $e \Phi\left(x_{g c}\right)$ to $\epsilon_{\max }$. We find

$$
n_{i}\left(x_{g c}\right)=n_{0}\left[e^{-\epsilon \Phi / T_{i}}-\left(1+\frac{\epsilon_{\max }-e \Phi}{T_{i}}\right) e^{-\epsilon_{\max } / T_{i}}\right]\left[1-\left(1+\frac{\epsilon_{\max }}{T_{i}}\right) e^{-\epsilon_{\max } / T_{i}}\right]^{-1}
$$

Taking $x_{g c} \approx x$ and setting the right hand side of Eq.(43) equal to the electron density $n_{e}(x)$ gives the potential $\Phi(x)$ in the Bohm presheath. Although $\Phi(x)$ is the solution to a 
transcendental equation which cannot be expressed in closed form, we can express $x$ as a simple algebraic function of $\Phi$

$$
x=R_{p}\left[1-\left(\frac{\epsilon_{\max }}{T_{i}}+1\right) e^{-\epsilon_{\max } / T_{i}}\right]^{-1 / 2}\left(1-e^{-e \Phi / T_{i}}-\frac{e \Phi}{T_{i}} e^{-\epsilon_{\max } / T_{i}}\right)^{1 / 2}
$$

Figure 4 shows a plot of $\Phi(x)$ and $n(x)$ for $\epsilon_{\max } / T_{i}=3$, using Eqs.(39) and (44). The plot would look identical to the naked eye for any $\epsilon_{\max } / T_{i}>2.5$. This $\Phi(x)$ has infinite slope at the wall, but of course it is valid only for $x$ more than a few ion Larmor radii away from the wall, where $x_{g c} \approx x$ is a good approximation, and for $x$ much more than a Debye length from the wall, so that $n_{e}=n_{i}$ is a good approximation to Poisson's equation.

Figure 5 shows the potential $\Phi(x)$ for a 1-D particle simulation with spatial diffusion artificially added by a Monte Carlo collision operator, as in Fig. 3, but in this case the Monte Carlo collision frequency was made great enough so that the spatial diffusion rate was much greater than the energy diffusion and energy loss rates. For comparison, the theoretical $\Phi(x)$ from Eq. (44) is also shown. The theoretical $\Phi(x)$ is in good agreement with the simulation result, except within a few Debye lengths of the wall, since the theoretical $\Phi(x)$ did not include finite Debye length, but assumed quasineutrality everywhere. The densities $n_{e}(x)$ and $n_{i}(x)$ found in the simulation are virtually identical to the parabolic profile given by Eq. (39), except within a Debye length or so of the wall, where they are both much lower, especially $n_{i}(x)$. (The relevant Debye length here is not the Debye length in the center of the plasma, but the edge Debye length $\delta x=\left(R_{p} / 2\right)^{1 / 3} \lambda_{D}^{2 / 3}$, for which the Debye length is equal to $\delta x$ at a distance $\delta x$ from the wall.)

\section{Finite Ion Larmor Radius}

We now consider how $\Phi(x)$ will be modified for $x$ within an ion Larmor radius of the wall, in the "magnetic presheath." The equations of motion for an ion in a uniform magnetic field $B_{0}$ and a potential $\Phi(x)$ are

$$
\begin{gathered}
\frac{d v_{x}}{d t}=v_{y} \Omega_{i}-\frac{e}{m_{i}} \frac{d \Phi}{d x} \\
\frac{d v_{y}}{d t}=-v_{x} \Omega_{i}
\end{gathered}
$$

where $\Omega_{i} \equiv e B_{0} / m_{i} c$ and $v_{x} \equiv d x / d t$. Integrating Eq.(46) over $t$ gives

$$
v_{y}=\left(x_{g c}-x\right) \Omega_{i}
$$


Figure 4

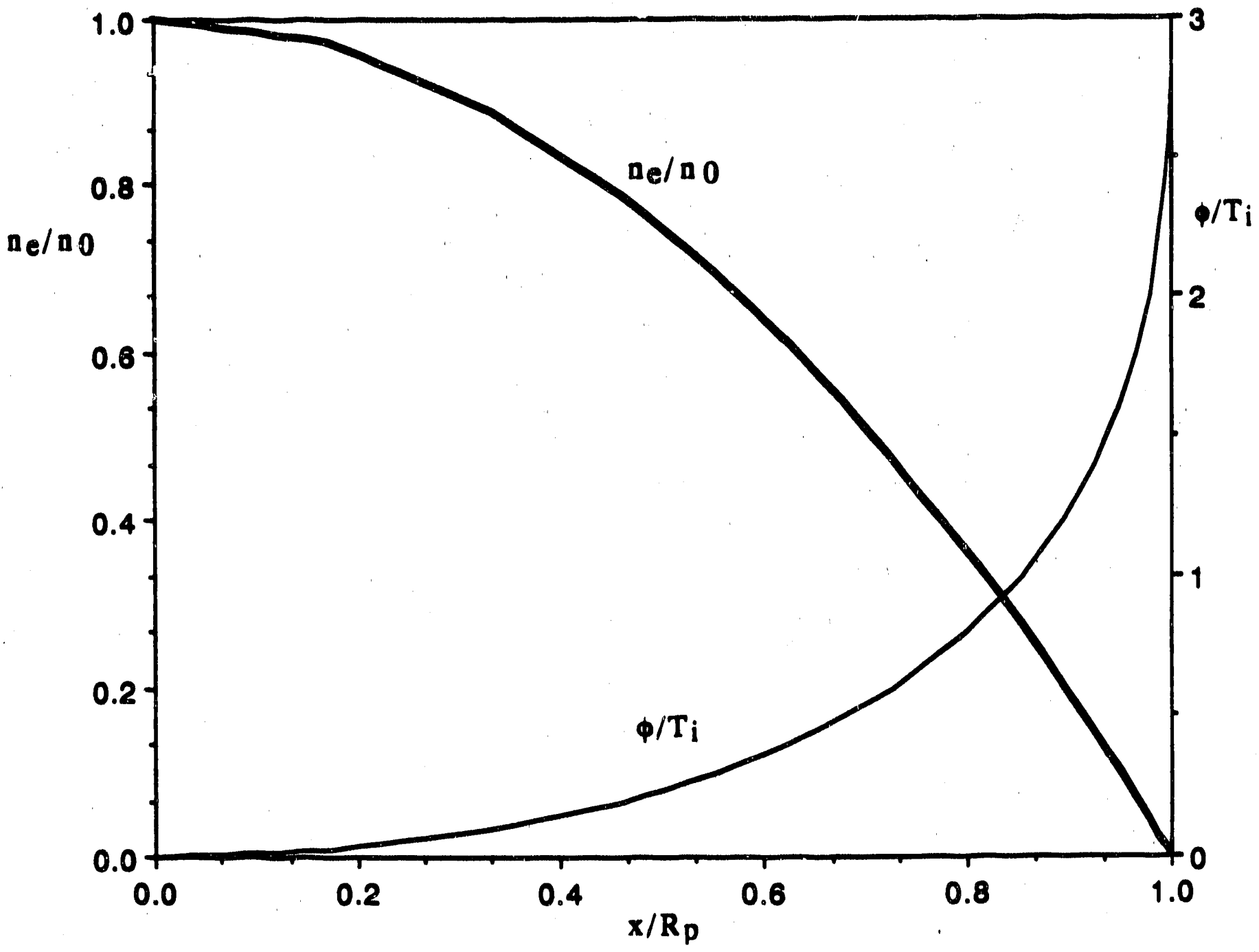

4. Density and potential profiles from Eqs.(39) and (44), valid when the spatial diffusion rate is greater than the energy diffusion rate. 
Flgure 5

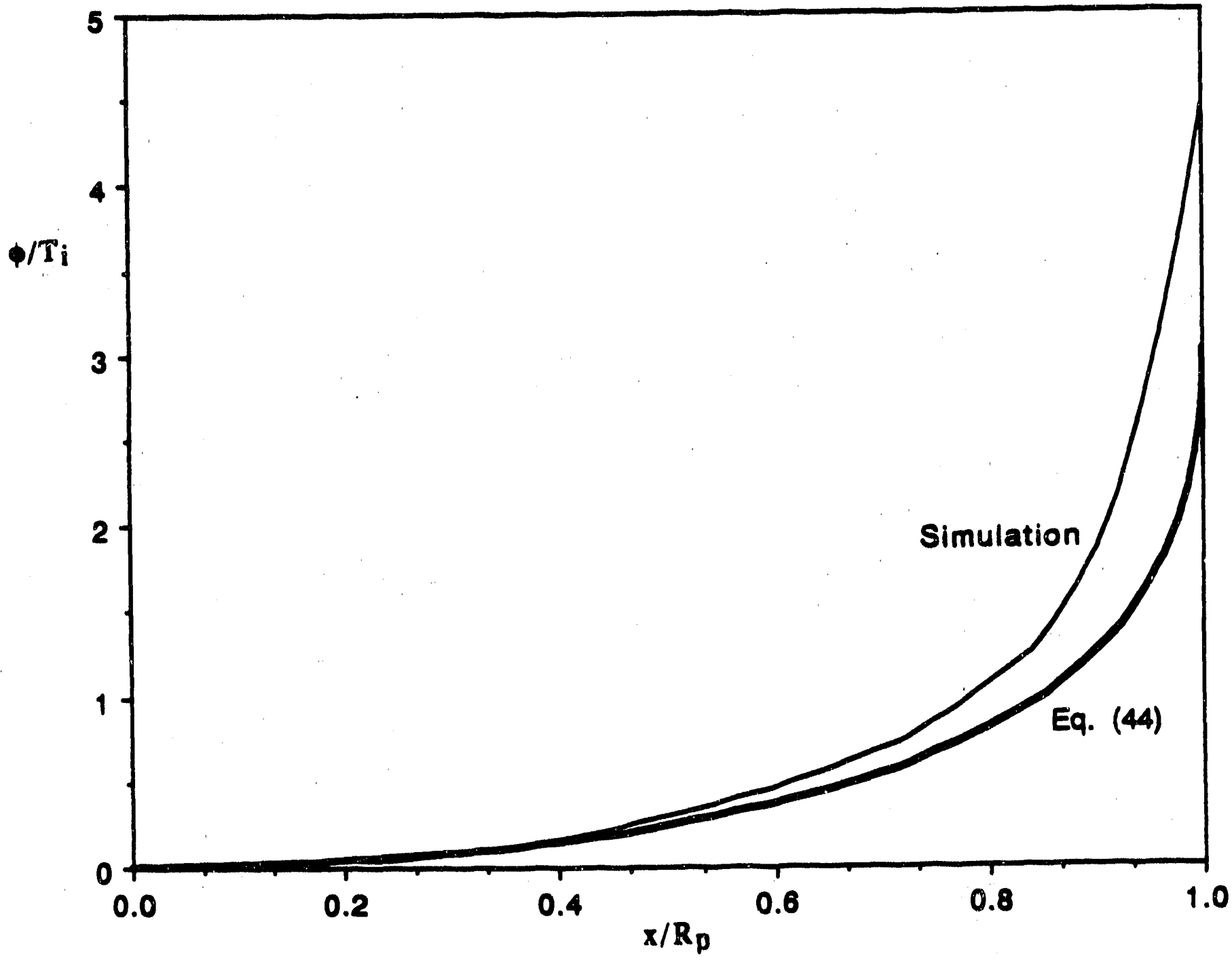

5. Potential profile from 1-D simulation with Monte Carlo diffusion of $x_{g c}$ and from Eq.(44). For the simulation the central Debye length is $0.018 R_{p}$, the edge Debye length is $0.055 R_{p}$, and the thermal ion Larmor radius is $0.18 R_{p}$. 
Putting Eq.(15) into Eq.(45), and iategrating over $x$ yields

$$
\frac{1}{2}\left(\frac{d x}{d t}\right)^{2}--\frac{1}{2}\left(x_{g c}-x\right)^{2} \Omega_{i}^{2}-\frac{e}{m_{i}} \Phi(x)+\frac{\epsilon}{m_{i}}
$$

In other words, the motion of dot in $x$ is described by an effective potential energy

$$
Q(g)=\frac{1}{2} m_{i}\left(x_{g c}-x\right)^{2} \Omega_{i}^{2}+e \Phi(x)
$$

Ions are lost to the wall when they reach the loss boundary in $\left(\epsilon, x_{g c}\right)$ space, given by

$$
\epsilon=Q\left(R_{p}\right)
$$

This loss boundary is a parabola with its minimum at $x_{g c}=R_{p}$ and $\epsilon=\epsilon_{\text {max }}$ (defined as in Sec. II), shown as a solid curve in Fig. 6. In the limit that diffusion of ions in $x_{g c}$ is much more rapid than diffusion in $\epsilon$, which we consider first, the ion distribution function $f_{i}\left(\epsilon, x_{g c}\right)$ must go nearly to zero at $\epsilon=\epsilon_{m a x}$, the lowest $\epsilon$ to intersect the loss boundary. In the opposite limit, $f_{i}\left(\epsilon, x_{g c}\right)$ would go to zero at the loss boundary. In addition to the loss boundary, there is another significant boundary in $\left(\epsilon, x_{g c}\right)$ phase space, the boundary of the non-physical region where particles cannot go because their effective potential energy $Q(x)$ is greater than their total energy $\epsilon$ for all $x$. This non-physical boundary $\epsilon=\epsilon_{\min }\left(x_{g c}\right)$ is shown by a dashed line in Fig. 6. It is calculated by simultaneously solving the equations

$$
\begin{gathered}
\frac{1}{2} m_{i}\left(x-x_{g c}\right)^{2} \Omega_{i}^{2}+e \Phi(x)-\epsilon_{\min }=0 \\
m_{i}\left(x-x_{g c}\right) \Omega_{i}^{2}+e \frac{d \Phi}{d x}(x)=0
\end{gathered}
$$

for the two unknowns $\epsilon_{\min }$ and $x$. (The curve for $\epsilon_{\min }\left(x_{g c}\right)$ shown in Fig. 6 was computed using the $\Phi(x)$ satisfying Eq.(44), which as we will show a posteriori is correct even when finite ion Larmor radius is taken into account, in the limit that the ion spatial diffusion rate is much greater than the energy diffusion rate. In the opposite limit, the $\Phi(x)$ given by Eq. (37) has to be corrected for finite ion Larmor radius, so an iterative procedure would have to be used to find $\epsilon_{\min }\left(x_{g c}\right)$, but it would still qualitatively resemble the curve plotted in Fig. 6.) Ions in the loss region of Fig. 6 are lost to the wall in in gyroperiod. Ions in the confined region, between the two curves, are confined for a radial diffusion tirne if they have $\epsilon>\epsilon_{\max }$ (which is equal to $3 T_{i}$ in Fig. 6), and for a (much longer) energy diffusion time if they have $\epsilon<\epsilon_{\max }$. Ion orbits for the points ir. phase space labelled $\mathrm{A}$, $\mathrm{B}$, and $\mathrm{C}$ in Fig. 6 are shown in Fig. 7. 
Figure 6

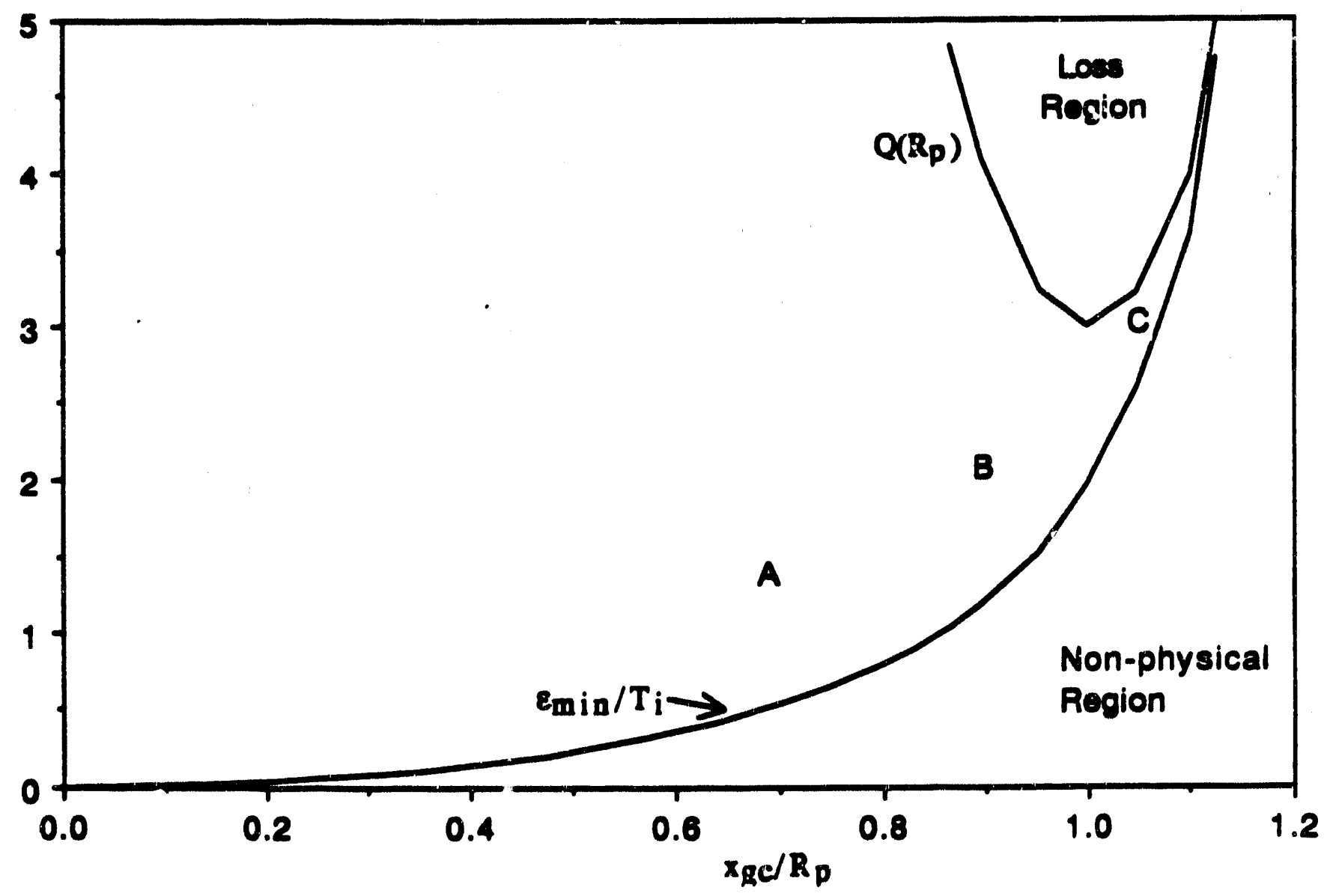

6. Ion phase space for finite Larmor radius $\rho_{i}=0.1 R_{p}$, showing the loss boundary given by Eq. (50) and $\epsilon_{\min }$ given by Eq. (51). 


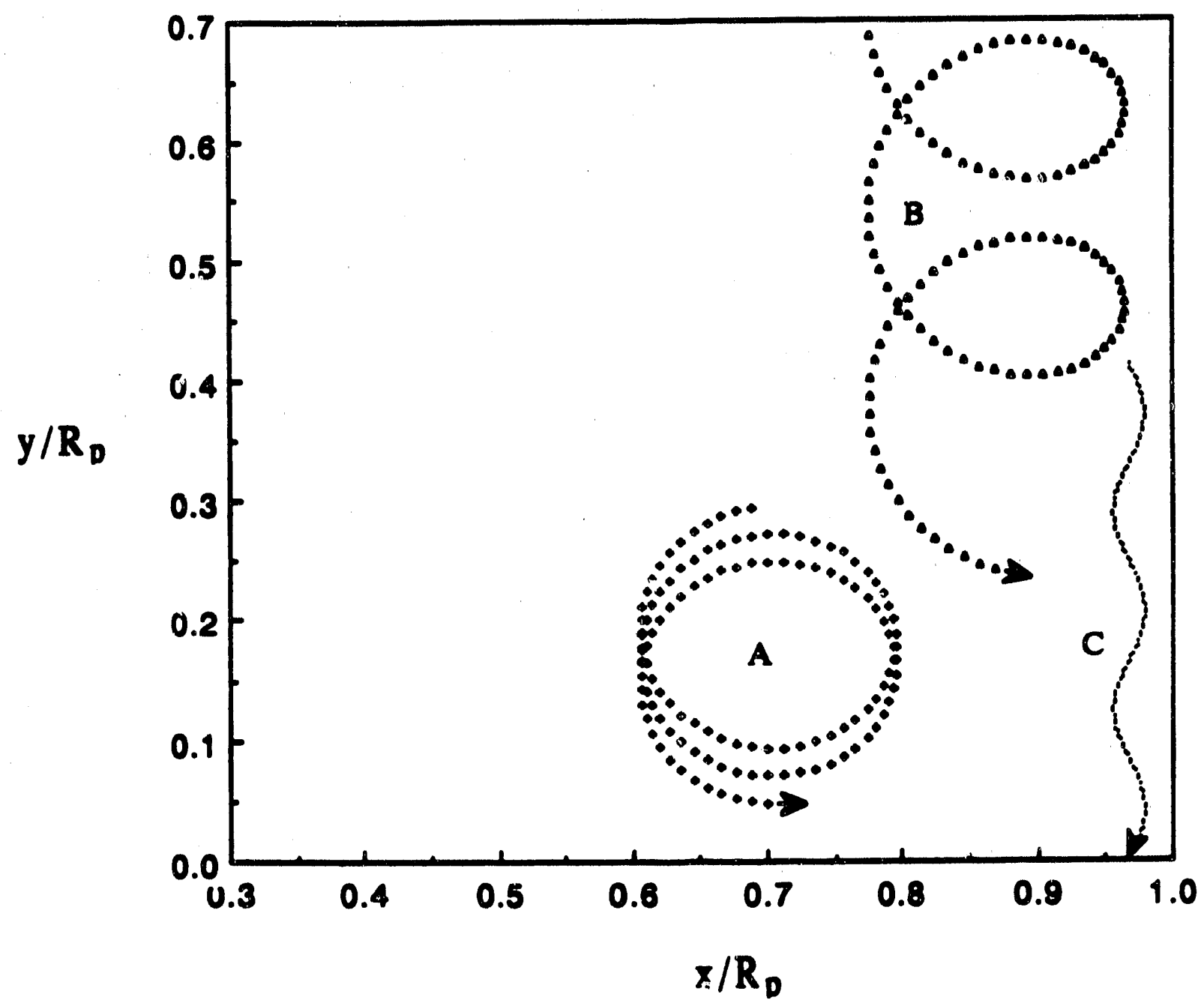

7. Ion orbits for the points in phase space labelled A, B, and C in Fig. 6. 
The ion density $n_{i}(x)$ is given by

$$
n_{i}(x)=\int_{-\infty}^{+\infty} d x_{g c} \int_{e \Phi(x)+\frac{1}{2} m_{i}\left(x-x_{g c}\right)^{2} \Omega_{i}^{2}}^{+\infty} d \epsilon \frac{f_{i}\left(\epsilon, x_{g c}\right)}{\left[\epsilon-e \Phi(x)-\frac{1}{2} m_{i}\left(x-x_{g c}\right)^{2} \Omega_{i}^{2}\right]^{1 / 2}}
$$

In the case we are considering, with ion spatial diffusion rate much greater than energy diffusion rate, a reasonable guess for the ion distribution function which is valid even for $x_{g c}$ within a Larmor radius of the wall is

$$
f_{i}\left(\epsilon, x_{g c}\right)=F(\epsilon)
$$

where $F(\epsilon)$ is still given by Eq. (42). Because $f_{i}\left(\epsilon, x_{g c}\right)$ has no explicit dependence on $x_{g c}$, the $x_{g e}$ integral in Eq.(20) can be put inside the $\epsilon$ integral, with the lower limit of the $\epsilon$ integral replaced by limits on the $x_{g c}$ integral, $x-\left[2(\epsilon-e \Phi) / m_{i}\right]^{1 / 2} \Omega_{i}^{-1}<x_{g c}<$ $x+\left[2(\epsilon-e \Phi) / m_{i}\right]^{1 / 2} \Omega_{i}^{-1}$. The $x_{g c}$ integral is then of the form $\int_{-a}^{+a} d x_{g c}\left[x_{g c}-a\right]^{-1 / 2}=\pi$, with no dependence on $\epsilon$, and Eq.(52) just reduces to Eq.(43), the expression we used for $n_{i}(\boldsymbol{x})$ neglecting finite ion Larmor radius. In other words, Ey.(43) for $\boldsymbol{n}_{i}(\boldsymbol{x})$ and Eq.(44) for $\Phi(x)$ are valid to all orders of finite ion Larmo: radius, in the limit that the ion spatial diffusion rate is much greater than the energy diffusion rate, so that $f_{i}\left(\epsilon, x_{g c}\right)$ has no explicit $x_{g c}$ dependence.

In the other limit, where the ion spatial diffusion rate is much less than the energy diffusion and loss rates, a reasonabie guess for $f_{i}\left(\epsilon, x_{g c}\right)$ is

$$
f_{i}\left(\epsilon, x_{g c}\right)=\exp \left(-\epsilon / T_{i}\right)-\exp \left(-Q\left(R_{p}\right) / T_{i}\right)
$$

for $\epsilon<Q\left(R_{p}\right)$, and $f_{i}\left(\epsilon, x_{g c}\right)=0$ for $\epsilon \geq Q\left(R_{p}\right)$. Here $Q\left(R_{p}\right)$, which is a function of $x_{g c}$, is given by Eq.(49). For $x-x_{g c}$ more than a few thermal ion Larmor radii from the wall, $Q\left(R_{p}\right) \gg T_{i}$, so, to excellent approximation, $f_{i} \approx \exp \left(-\epsilon / T_{i}\right)$. For $x$ more than a few thermal ion Larmor radii from $x_{g c}$, the lower limit of the $\epsilon$ integral in Eq.(52) is much greater than $T_{i}$, so these $x_{y c}$ do not contribute significantly to $n_{i}(x)$, and it follows that for $x$ more than a few thermal ion Larmor radii from the wall, $f_{i} \approx \exp \left(-\epsilon / T_{i}\right)$. Then $f_{i}\left(\epsilon, x_{g c}\right)$ has no explicit $x_{g c}$ dependence, and the expressions we derived before for $n_{i}(x)$ and $\Phi(x)$ are valid. Within a few ion Larmor radii of the wall, the $x_{g c}$ dependence of $f_{i}\left(\epsilon, x_{g c}\right)$ must be retained. Then, for $x$ near $R_{p}$, but many ion Larmor radii from $-R_{p}$,

$$
n_{i}(x)=\int_{-\infty}^{+x_{\text {max }}} d x_{y c} \int_{e \Phi(x)+\frac{1}{2} m_{i}\left(x-z_{g c}\right)^{2} \Omega_{i}^{2}}^{Q\left(R_{p}\right)} d \epsilon \frac{\exp \left(-\epsilon / T_{i}\right)-\exp \left(-Q\left(R_{p}\right) / T_{i}\right)}{\left[\epsilon-e \Phi(x)-\frac{1}{2} m_{i}\left(x-x_{g c}\right)^{2} \Omega_{i}^{2}\right]^{1 / 2}}
$$


where $x_{\max }$ is the $x_{g c}$ at which the upper and lower limits of the $\epsilon$ integral are equal

$$
x_{\text {mex }}=\frac{1}{2}\left(x+R_{p}\right)+\frac{e\left[\Phi\left(R_{p}\right)-\Phi(x)\right]}{m_{i} \Omega_{i}^{2}\left(R_{p}-x\right)}
$$

Then, doing the $\epsilon$ integration,

$$
\begin{aligned}
& n_{i}(x)=n_{0} \exp \left(\frac{-e \Phi}{T_{i}}\right) \int_{-\infty}^{+x_{m a x}} d x_{g c}\left(\frac{m_{i}}{2 \pi T_{i}}\right)^{1 / 2} \Omega_{i} \\
& {\left[\exp \left(\frac{-m_{i}\left(x-x_{g c}\right)^{2} \Omega_{i}^{2}}{2 T_{i}}\right) \operatorname{erf}\left(\sqrt{\frac{2 m_{i} \Omega_{i}^{2}\left(R_{p}-x\right)\left(x_{\max }-x_{g c}\right)}{T_{i}}}\right)-\right.} \\
& \left.\frac{2}{\sqrt{\pi}} \exp \left(\frac{-e\left(\Phi\left(R_{p}\right)-\Phi\right)}{T_{i}}\right) \exp \left(\frac{-m_{i}\left(R_{p}-x_{g c}\right)^{2} \Omega_{i}^{2}}{T_{i}}\right) \sqrt{\frac{2 m_{i} \Omega_{i}^{2}\left(R_{p}-x\right)\left(x_{\max }-x_{g c}\right.}{T_{i}}}\right]
\end{aligned}
$$

The integral is nearly equal to 1 when the argument of the erf is much greater than 1 , since in this case the erf is nearly 1 , and the second term in the integral is exponentially small. This is true to excellent approximation whenever $x$ is more than a few Larmor radii from the wall, and in this case $n_{i}(x)$ is hardly affected by finite ion Larmor radius. Even if $\boldsymbol{x}$ is within an ion Larmor radius of the wall, but much more than a Debye length from the wall, the argument of the erf will not be much less than 1 , because $\Phi(x)$ will be at least $T_{i}$ below the wall potential (since most of the rise in potential takes place within a few Debye lengths of the wall). In this case, the integral will not be reduced by more than a factor or 2 or so, so $n_{i}(x)$ will be within a factor of 2 of the value that it would have, at that $\Phi(x)$, if finite :on Larmor radius were neglected. Hence the self-consistent $\Phi(x)$ in this region, including the effect of finite ion Larmor radius, will only be a fraction of $T_{i}$ above the value it would have if finite ion Larmor radius were neglected, and it will still be true that most of the rise in potential (which we assume is a few times $T_{i}$ ) will occur within a few Debye lengths of the wall. The width of the sheath, where most of the rise in potential takes place, might be increased by a factor of 2 or so as a result of finite ion Larmor radius, but it will still be comparable to a Debye length, even if a Debye length is much narrower than an ion Larmor radius. The magnetic presheath will only have a modest effect on the potential profile, raising it sume fraction of $T_{i}$ within an ion Larmor radius of the wall.

In Fig. 8 we show the potential profile $\Phi(x)$ for two simulations with the same Debye length but with ion Larmor radius differing by about a factor of 2 . In one case the thermal ion Larmor radius was $0.082 R_{p}$ and in the other case it was $0.154 R_{p}$. The Monte Carlo 


\section{Floure 8}

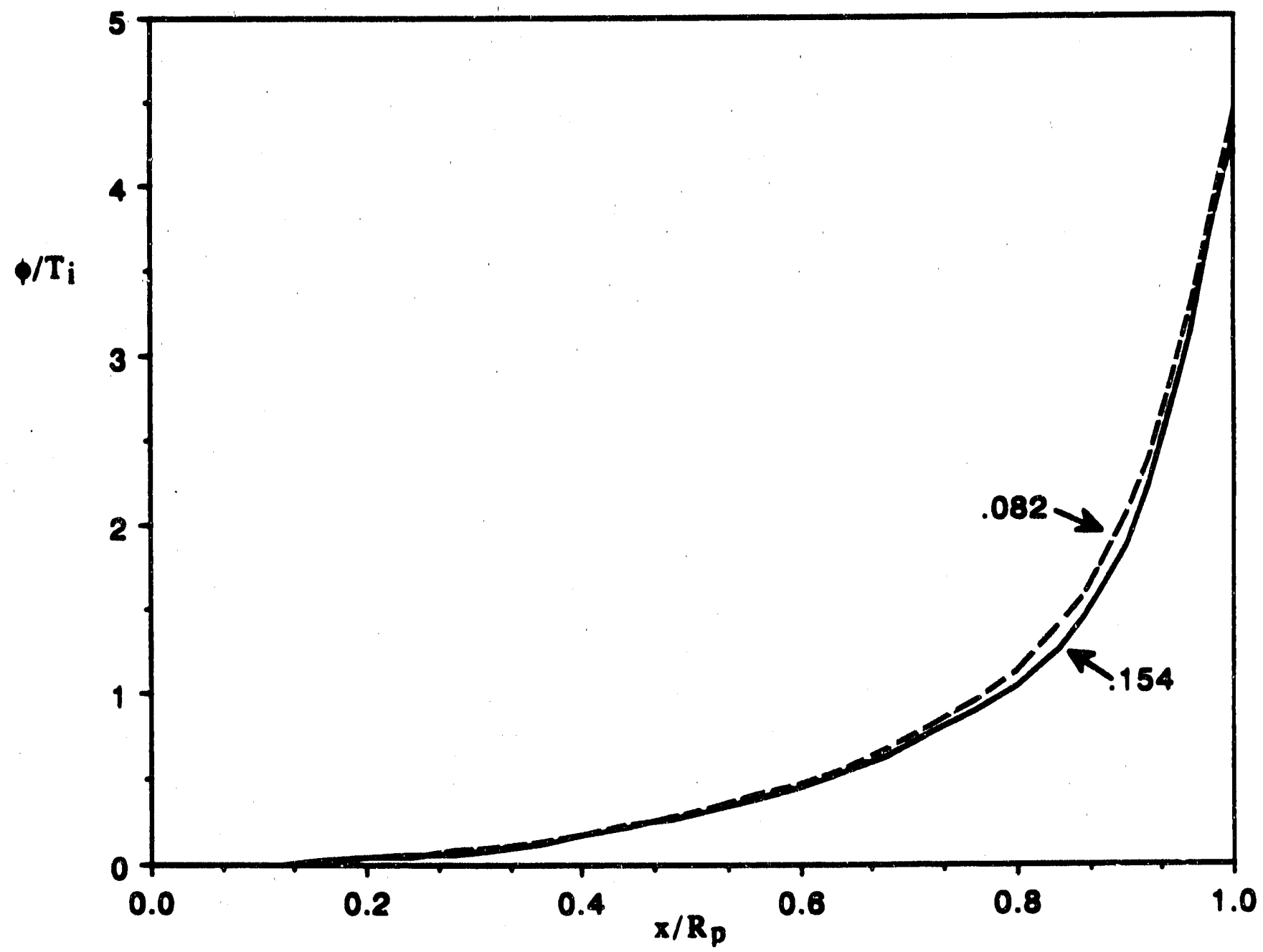

8. 1-D simulations with Monte Carlo diffusion in $x_{g c}$, for two different values of the thermal ion Larmor radius, $0.154 R_{p}$ and $0.082 R_{p}$. For both simulations the central Debye length is $0.022 R_{p}$ and the edge Debye length is $0.065 R_{p}$. 
ion collision frequency is great enough to make the spatial diffusion rate greater than the energy diffusion rate, and, as expected in this limit, the potential profile is nearly independent of ion Larmor radius. The potential profiles do not differ by more than $0.2 T_{i}$ at any $x$, despite the factor of 2 difference in ion Larmor radius. The small differences that do exist in the potential profile (due to the fact that the energy diffusion rate is not completely negligible compaced to the spatial diffusion rate) are greatest within an ion Larmor radius of the wall, the region of the magnetic presheath. In order to show that the scale length of the potential near the wall is comparable to tine Debye length, we show potential profiles for two simulations with Debye length differing by about a factor of 2 , in Fig. 9. The Debye lengths in the center of the plasma are $0.018 R_{p}$ and $0.037 R_{p}$ in these two simulations. In this case, in contrast to Fig. 8, there is dramatic difference in the scale length of the potential near the wall, with the scale length being comparable to the edge Debye length, $\delta_{z}=\left(R_{p} / 2\right)^{1 / 3} \lambda_{D}^{1 / 3}$ in each simulation.

In a fully 2-D simulation, as opposed to a 1-D simulation with a Monte Carlo collision operator, there is reuson to believe that the scale length of the sheath at the wall will be an ion Larmor radius, rather than a Debye, if $\omega_{p i} \gg \Omega_{i}$, i.e. if a Debye length is much less than an ion Larmor radius at a distance of an ion Larmor radius from the wall. Unfortunately such a 2-D simulation would be very expensive, because of the great number of particles required, and we have not, done such a simulation. The 2-D simulation shown in Fig. 2, for example, has Debye lengih comparable to ion Larmor radius at a distance of one ion Larmor radius from the wall, although the Debye length would be much less than this at the higher densities in the middle of the plasma. This conjecture has therefore not been demonstrated.

The reason for this expectation is the drag force $F_{y}$ in the $y$ direction which can occur in a 2-D simulation, and which would cause particles to drift at a velocity $e F_{y} / c B_{0}$ in the $x$ direction. Such a drag force can result either from collisions or from a two-stream instability when the ions and electrons have different drift velocities in the $y$ direction. It can also result from a Kelvin-Helmholtz instability ${ }^{9}$ when the drift velocity of the ions in the $y$ direction varies too sharply with $x$, or from a lower hybrid drift instability ${ }^{10}$ when the density gradient is too steep.

The electrons at a given $x$ will have a ruean $v_{y}$ (if we neglect finite electron Larmor radius) of

$$
u_{e}(x)=\frac{c}{B_{0}} \frac{d \Phi}{d x}-\frac{v_{e}^{2}}{\Omega_{e}} \frac{1}{n} \frac{d n}{d x}
$$


Figure 9

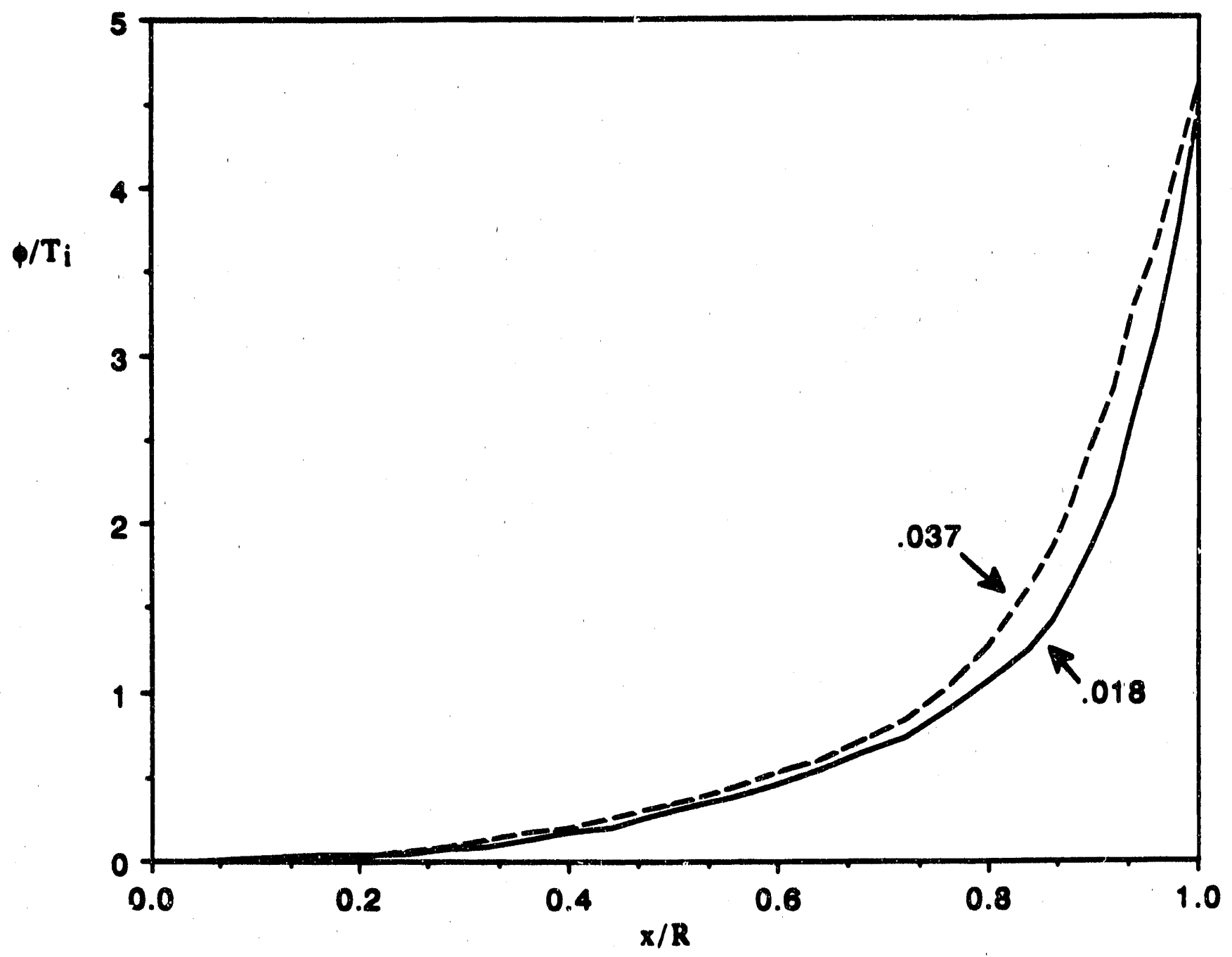

9. 1-D simulation with Monte Carlo diffusion in $x_{g c}$, for two different values of the Debye length. The central Debye lengths are $0.018 R_{p}$ and $0.037 R_{p}$, and the edge Debye lengths are $0.055 R_{p}$ and $0.091 R_{p}$. 
while the ions will have a mean $v_{y}$ that depends on a weighted average of $d \Phi / d x$ and $d n / d x$ (as well as higher derivatives) within an ion Larmor radius of $x$

$$
u_{i}(x)=\frac{\Omega_{i}}{n(x)} \int_{-\infty}^{+\infty} d x_{g c} \int_{e \Phi(x)+\frac{1}{2} m_{i}\left(x-x_{g c}\right)^{2} \Omega_{i}^{2}}^{+\infty} d \epsilon \frac{\left(x-x_{g c}\right) f_{i}\left(\epsilon, x_{g c}\right)}{\left[\epsilon-e \Phi(x)-\frac{1}{2} m_{i}\left(x-x_{g c}\right)^{2} \Omega_{i}^{2}\right]^{1 / 2}}
$$

As shown previously, the potential $\Phi(x)$ obtained using a Monte Carlo collision model for radial transport (ignoring drag in the $y$ direction) rises on a scale length small compared to an ion Larmor radius when $x$ is much less than a Larmor radius from the wall. The density given by Eq.(39) also has a scale length much shorter than a Larmor radius when $x$ is much less than a Larmor radius from the wall. This means that $u_{e}$ will be much greater than the ion thermal velocity $v_{i}=\left(T_{i} / m_{i}\right)^{1 / 2}$ when $x$ is much less than a Larmor radius from the wall, due to both the $d \Phi / d x$ and $d n / d x$ terms in Eq.(58). The ion drift relority $u_{i}$ is of course never much greater than $v_{i}$. This difference in the local drift velocities $u_{e}$ and $u_{i}$ would give rise to a collisional drag force $F_{y}$ (and perhaps a much greater drag force due to modified two-stream instability) of equal and opposite sign on the ions and electrons, which would cause both the ions and electrons to drift toward the wall with a velocity $e F_{y} / c B_{0}$. About an ion Larmor radius, away from the wall or a little more, the local ion drift velocity $u_{i}(x)$ will be greater than $u_{e}(x)$ because the ions would pick up a contribution from the very steep $d \Phi / d x$ near the wall, while the electrons will only see the local $d \Phi / d x$. In this region the drag forces will be in the other direction, and will cause both the ions and electrons to drift away from the wall. These radial drifts should be included in the terms $V_{i}$ and $V_{e}$ which appear in Eqs.(23) and (24). The result will be to modify $n(x)$ and $\Phi(x)$ is such a way as to decrease the drag forces.

Although the drag forces make equal contributions to $V_{e}$ and $V_{i}$, the contribution to $V_{e}$ will have a much greater effect on $f_{e}$ than the contribution to $V_{i}$ will have on $f_{i}$, because the dominant terms in Eq.(23) are much greater than any of the terms in Eq.(24). If the contribution of the drag force to $V_{e}$ is competitive with the electron spatial diffusion and source terms in Eq.(36) or (38), then $n(x)$ and $\Phi(x)$ will be substantially modified, and the drag forces substantially reduced. But at this magnitude of drag forces, the terms in the ion equation, Eq.(23), will hardly be affected by the drag force contribution : $V_{i}$. It follows that the drag forces will never have much effect on the ion equation, because they will be self-consistently limited in magnitude by their effect on the electron equation. Equation (23), and the equations that follow from it, such as Eqs.(34), (43), and (57), will still be valid if there are drag forces present, but Eq.(39) will be modified. 
We consider an artificially simple model for drag which illustrates the effect it has on $n(x)$ and $\Phi(x)$, and how this effect may be calculated. We suppose that the drag forces are infinite if $d^{2} \Phi / d x^{2}$ exceeds some critical value

$$
\frac{d^{2} \Phi}{d x^{2}}>\Phi_{c r i t}^{\prime \prime}
$$

and zero otherwise. If we take $\Phi_{\text {crit }}^{\prime \prime}$ to be of order $T_{i} / e \rho_{i}^{2}$, then this is roughly what would be expected from a two-stream instability that turned on suddenly when $\left|u_{e}-u_{i}\right|>v_{i}$, although the real condition would involve a complicated integral functional of $\Phi(x)$ rather than just the second derivative. With this model, there must be an $x_{0}$ such that $d^{2} \Phi / d x^{2}<$ $\Phi_{\text {crit }}^{\prime \prime}$ for $|x|<x_{0}$ and $d^{2} \Phi / d x^{2}=\Phi_{c r i t}^{\prime \prime}$ for $|x|>x_{0}$. Both $\Phi(x)$ and $d \Phi / d x$ are continuous across $x= \pm x_{0}$. For $|x|<x_{0}$, the drag force is zero, and Eq.(38) is still satisfied. Equation (39) is generalized to

$$
n(x)=n_{0}-\frac{S x^{2}}{2 D_{x, e}}
$$

which still satisfies Eq.(38). It is no longer true that $n_{0}=R_{p}^{2} S / D_{s, e}$, because Eq.(38) is no longer satisfied all the way to the wall, only out to $x= \pm x_{0}$, but $n_{0}$ will be close to $R_{p}^{2} S / D_{x, e}$ if $x_{0}$ is close to the wall, and Eq.(39) will be a good approximation to $n(x)$ for $\boldsymbol{R}_{p}-|\boldsymbol{x}| \gg R_{p}-x_{0}$. In this region $\Phi$ is still given in terms of $n$ by Eq.(57), or by Eq.(34) which is a good approximation to Eq.(57), or by Eq. (43). Note that the resulting expression for $\Phi(x)$ will involve two unknowns, $n_{0}$ and $\epsilon_{\max }$, which will not necessarily be the same as in the case where there is no drag force, because the electron loss rate is not the same. For $|x|>x_{0}, \Phi(x)$ is given by

$$
\Phi(x)=\Phi\left(x_{0}\right)+\left.\left(|x|-x_{0}\right) \frac{d \Phi}{d x}\right|_{x=x_{0}}+\frac{1}{2}\left(|x|-x_{0}\right)^{2} \Phi_{c r i t}^{\prime \prime}
$$

where $\Phi\left(x_{0}\right)$ and $d \Phi / d x$ evaluated at $x=x_{0}$ are given by the solution in the region where $|x|<x_{0}$. The density $n(x)$ is then given by Eq.(57), or more simply and to good approximation, by Eq.(34), or by Eq. (43). The drag force on the electrons, which can have any value between zero and infinity when $d^{2} \Phi / d x^{2}=\Phi_{c r i t}^{\prime \prime}$, adjusts itself so that Eq.(24) is satisfied with this $n_{e}(x)$. The required drag force will be such that the additional term in $V_{e}$ due to the drag force makes the $V_{e}$ term comparable to the other dominant terms in Eq.(24), viz. those that have been kept in Eq.(38). The two unknowns, $n_{0}$ and $\epsilon_{\max }$, may be found by solving two equations, ore equating the electron loss rate with the integrated source, and the other equating the ion loss rate with the electron loss rate. If $x_{0}$ is near the 
wall, as it will be if $R_{p} \gg \rho_{i}$ and $\Phi^{\prime \prime} \approx T_{i} / e \rho_{i}^{2}$, then $n_{0}$ and $\epsilon_{\max }$ will be close to the values they have when there is no drag force. To illustrate the effect of the drag force on $n(x)$ and $\Phi(x)$, we have plotted these quantities in Fig. 10 for the case where $\Phi_{c r i t}^{\prime \prime}=100 T_{i} / e R_{p}^{2}$ (appropriate for $\rho_{i} \approx 0.1 R_{p}$, , assuming that $\epsilon_{\max }$ and $n_{0}$ are unchanged by the drag force, and using Eq.(43) to relate $\Phi(x)$ to $n(x)$. The curves for $\Phi(x)$ and $n(x)$ in the absence of drag force are shown as dashed curves in Fig. 10, for comparison; these are identical with the curves in Fig. 4. For these parameters, we find

$$
\begin{aligned}
x_{0} & =0.83 R_{p} \\
\Phi\left(x_{0}\right) & =0.93 T_{i} / e \\
\left.\frac{d \Phi}{d x}\right|_{y=x_{0}} & =\frac{3.5 T_{i}}{e R_{p}}
\end{aligned}
$$

The effect of the drag force is to broaden the sheath (decreasing $d \Phi / d x$ near the wall but increasing it just to the right of $x_{0}$ ), and to decrease the density in the region $x_{0}<x<R_{p}$ where the drag force is present due to a marginal two-stream instability. The scale length of the rise in potential near the wall is now on the order of $\rho_{i}$. This would be true even if the drag force were due to collisions, rather than to a hypothetical instahility which turned on at a certain value of $d^{2} \Phi / d x^{2}$, as long as the collision frequency were at least as great as the the frequency of collisions causing the spatial electron diffusion $D_{x, e}$. (Such a model would make the calculation more difficult, of course.)

\section{Summary and Conclusions}

Important differences exist between 1-D and 2-D simulations of magnetized plasmas, and these differences have been illustrated for the case of a plasma slab in a uniform magnetic field in the $z$ direction, bounded by conducting absorbing walls at $x= \pm R_{p}$, so that the magnetic field is parallel to the walls. This model has some relevance to the edge region of a tokamak. ${ }^{1}$ If the simulation starts with a uniform density of ions and electrons, then initially particles within a Larmor radius of the wall can be lost, and there are more ions than electrons in this class. This almost immediately results in the plasma potential becoming negative with respect to the wall, in order to hoid in ions, and the electric field is limited to rarrow sheaths near the wall, with widths comparable to either the Debye length or the electron Larmor radius, whichever is greater. In a $1-D$ simulation there are no forces exerted in the $y$ direction, so there can be no changes in the $x$ guiding center position of a particle. The plasma then remains in this state, with nearly uniform 
Figure 10

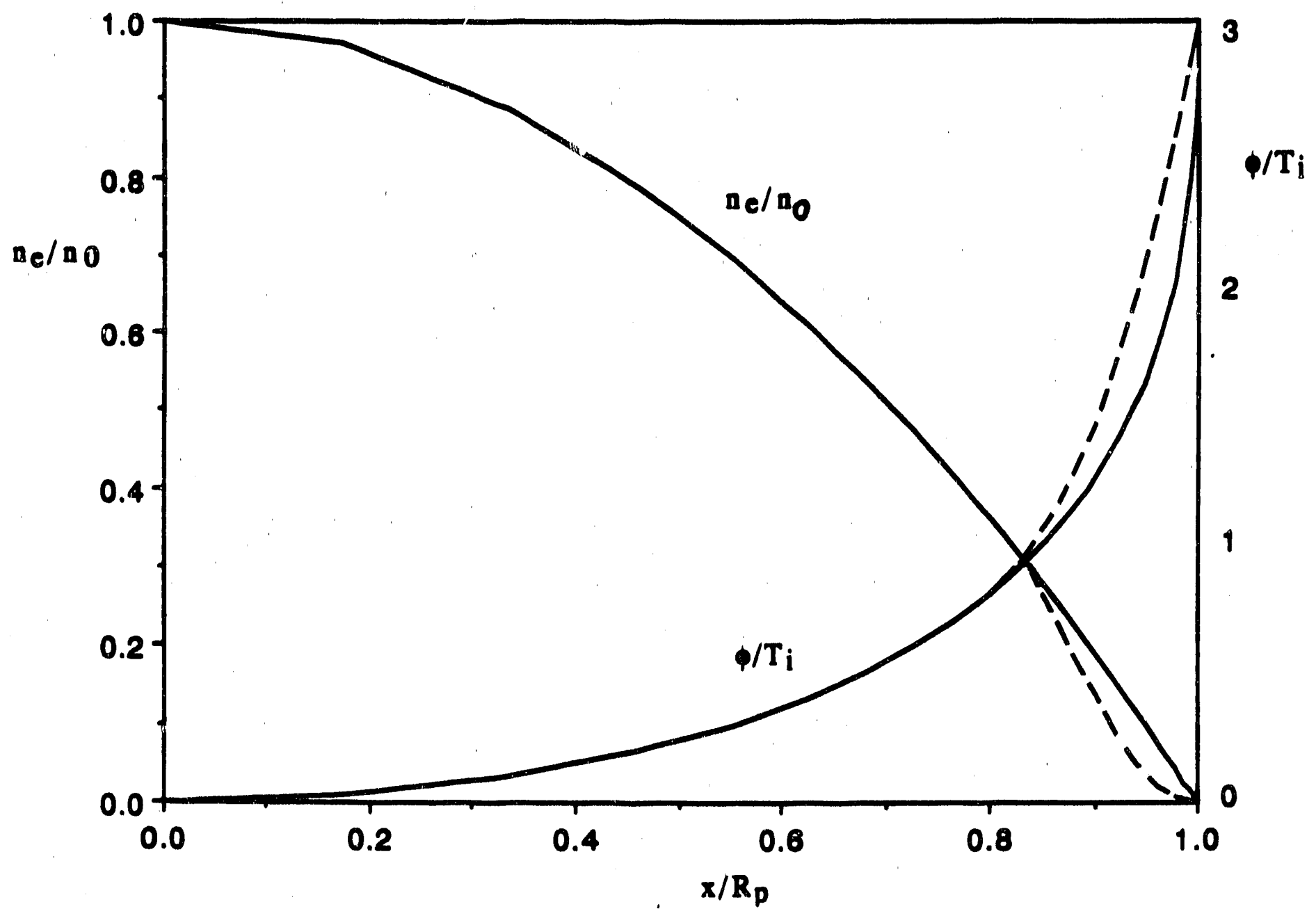

10. Effect of drag on edge potential and density, for $\Phi_{c r i t}^{\prime \prime}=100 T_{i} e / R_{p}^{2}$. Potential profile from Eq.(62) is the dashed line, and potential profile from Eq. (44), with nn drag, is the solid line. The corresponding densities, also shown by dashed and solid lines, are related to the potentials by Eq.(43). 
density except in the sheaths. Further losses of particles to the walls are possible only if particles can diffuse up in energy so that their Larmor radius is comparable to $R_{p}$, and this process will be exponentially slow if the thermal ion Larmor radius is much less than $R_{p}$. In a 2-D simulation, by contrast, particles can diffuse to the walls as a result of collisions, or interractions with waves, which change $x_{g c}$. The density profile will be governed by the diffusion of the more slowly diffusing species, electrons in our case. If the electron diffusion coefficient is spatially uniform, which seems to be a good approximation in the 2-D simulations we have done, then the density profile will be parabolic, going almost to zero at the walls. The effect of this diffusion can be incorporated in a 1-D simulation, without the expense of a fully 2-D simulation, by adding a phenomenological Monte Carlo diffusion of $x_{g c}$ for the particles. When this is done, the 1-D simulation evolves to an equilibrium that is in excellent agreement with the 2-D simulation, although a 2-D simulation is still needed in order to examine the details of the Kelvin-Helmholtz turbulence which is principally responsible for particle transport. ${ }^{8}$ An analysis has been made of the potential and density profiles that should evolve in these simulations, in different limits of the parameters. The ion density profile is also nearly parabolic, since the plasma must be quasineutral except in the sheaths near the walls. In the quasineutral Bohm presheath region, the potential follows a Boltzmann expression, given by Eq.(37), in the limit that the ion energy diffusion rate is much greater than the spatial diffusion rate to the wall. In the opposite limit, which would apply within an ion Larmor radius of the wall, and even further from the wall if there were more massive impurity ions causing enhanced collisional transport, the Boltzmann relation is somewhat modified, and the potential profile is given by Eq.(44). The non-neutral Debye sheaths near the walls, where much of the rise in potential occurs, have widths comparable to the greater of the Debye length and the electron Larmor radius, as in the 1-D model without diffusion. This is true even though the sheath width is much less than an ion Larmor radius. Equation (44) is correct in the limit for which it was derived, even when finite ion Larmor radius is taken into account. The potentipl profile given by Eq.(37) would have to be modified slightly, within an ion Larmor radius of the wall (the magnetic presheath region ${ }^{\theta}$ ), but the potential profile still hav the same qualitative appearance. These analytic results have been confirmed by 1-D simulations with Monte Carlo diffusion of $x_{g c}$. In addition to the diffusion in $x_{g c}$ that occurs in a 2-D simulation, there is another 2-D effect that should be important if the plasma has high enough density so that $\omega_{p i} \gg \Omega_{i}$ at a distance of an 
ion Larmor radius from the wall. This effect is the drag in the $y$ direction that would be exeried by the ions on the electrons within an ion Larmor radius of the wall, as a result of collisional drag or modified two-stream instabilities. This drag causes the electrons and ions to flow away from this region, until the scale length of the potential rise near the wall has been broadened to about an ion Larmor radius. We have not been able to confirm this conjecture with a 2-D simulation, because the simulation would have to have a large number of particles and would be very expensive. In any case $\omega_{p i}$ is typically not much greater than $\Omega_{i}$ near the edge of a tokamak, so this effect may not be important.

This drag force does not have much effect on the overall confinement time, because the instability (or collisions) causing the drag force is only important at the very edge of the plasma, within about an ion Larmor radius of the wall, while the source extends over the whole plasma. The drag force merely reduces the density in the narruw region where the instability is present, effectively moving the edge of the plasma in by about an ion Larmor radius from the wall, while the interior of the plasma is hardly affected. The situation would be different if the source were localized to the region near the wall where the instability was present. This might happen if the source were dominated by ionization of neutrals coming from the wall, which could not penetrate more than a few ion Larmor radii into the plasma. In this case the equilibrium density in the interior of the plasma would be substantially reduced as a result of the drag force, or the source term (and hence the flux to the wall) would have to be substantially increased to keep the interior density the same as it would be without the drag force. There is evidence that the improved confinement during $\mathrm{H}$-mode in JET is associated with a lower level of edge fluctuations, ${ }^{15}$ although these were probably microtearing or ballooning modes, not Kelvin-Helmholtz modes.

By using 1-D simulations with phenomenological Monte Carlo diffusion of $x_{g c}$, and perhaps phenomenological flow of $x_{g c}$ as well, it should be possible to reproduce the the macroscopic behavior of a 2-D simulation, much more cheaply. Such 1-D simulations could be used to make surveys of parameter space, and a few fully 2-D simulations could then be made to confirm the validity of the phenomenulogical terms, and to determine such details as the turbulent wave spectrum. This procedure should be applicable not only to the crossfield sheath in a plasma slab, but also to other physical situations that may be of more direct relevance to tokamak edge phenomena. It is likely that realistic modelling of tokamak edge transport will require $3-D$. models, which allow instabilities to have a spectrum of 
parallel wave numbers $k_{\|}$. At $k_{\|} v_{e}>\omega$, where $v_{e}=\left(T_{e} / m_{e}\right)^{1 / 2}$ is the electron thermal velocity and $\omega$ is the wave frequency, Kelvin-Helmholtz instrabilities and lower hybrid drift instabilities are supressed, but electron drift waves (including ${ }^{16}$ the universal instability, trapped electron instabilities, temperature gradient instabilities, and related dissipative instabilities) can be unstable at arbitrarily small density gradients. Finite $k_{\|} v_{e} / \omega$ also plays a role in the saturation of the rippling mode, ${ }^{17}$ a resistivity gradient driven mode which is thought to be important in tokomak edge regions characterized by high $n_{e}$ and a low $T_{e}$ which is a steep function of radius. The details of the density, temperature and current profiles at the plasma edge should determine the relative importance of such 3D effects compared to the 2-D instabilities appearing in the particle simulations we have described. Analytic models will be especially important in interpreting 3-D models of crossfield transport, because 3-D simulations are too expensive to allow extensive variation of parameters.

\section{Acknowledgments}

The authors would like to thank C. K. Birdsall and D. Holland for useful discussions, and one of us (M. J. G.) would also like to thank C. K. Birdsall for his hospitality at the Electronics Research Lab of the University of California at Berkeley, where part of this research was done. This research was supported by U. S. Department of Energy Contracts No. DE-AC02-78ET51013 and No. FG03-86ER53220. 


\section{References:}

1 R. D. Haseltine, "Self-Consistent Radial Sheath," in IFS Newsletter, Vol. VII, No. 2, December 1988

${ }^{2}$ K. Theilhaber, "ES2 User's Manual - Version 1," Memorandum No. UCB/ERL M87/23, Electronics Research Laboratory, College of Engineering, University of California at Berkeley, Mzy 11, 1987.

3 C. K. Birdsall and A. B. Langdon, Plasma Physics via Computer Simulation (McGraw Hill, New York, 1985), p. 308.

4 W. Nevins, Y. Matsuda, and M. Gerver, J. Comp. Phys. 39, 226 (1981).

5 U. Daybelge and B. Bein, Phys. Fluids 24, 1190 (1981).

6 R. Chodura, Phys. Fluids 25, 1628 (1982).

${ }^{7}$ Lewi Tonks and Irving Langmuir, Phys. Rev. 34, 876 (1929); David Bohm, in The Characteristics of Electrical Discharges in Magnetic Fields, edited by A. Guthrie and R. K. Wakerling, McGraw-Hill, 1949

8 K. Theilhaber and C. K. Birdsall, Phys. Rev. Lett. 62, 772 (1989).

- W. Horton, T. Tajima, and T. Kamimura, Phys. Fluids 30, 3485 (1987); S. Chandrasekhar, Bydrodynamic and Hydromagnetic Stability (Clarendon Press, Oxford, 1961), Chapter 11; H. Helmholtz, "Ueber discontinuirliche Flüssigkeitsbewegungen," Wissenschaftliche Abhandlungen 146-57 (J. A. Barth, Leipzig, 1882), translated by Guthrie in Phil. Mag. (Ser. 4) 36, 337-46 (1868); Lord Kelvin, "Hydrokinetic solutions and observations," "On the motion of free solids through a liquid, 69-75," "Influence of wind and capillarity on waves in water supposed frictionless, 76-85," Mathematical and Physical Papers iv: Hydrodynamics and Genural Dynamics (Cambridge, England, 1910).

10 N. A. Krall and P. C. Liewer, Phys. Rev. A4, 2094 (1971).

${ }^{11}$ D. Holland, B. Fried, and G. Morales, Sherwood Controlled Fusion Theory Meeting, Gatlinburg, TN, April 18-20, 1988, paper 3D23.

12 K. Theilhaber and C. K. Birdsall, "Kelvin-Helmholtz Vortex Formation and Particle Transport in a Cross-Field Plasma Sheath I: Transient Behavior," to be published in Phys. Fluids (1989), Figure 13.

13 L. Boltzmann, Vorlesungen über die Gas Theorie (J. A. Barth, Leipzig, 1910), Ch. 1, F. 15 
14 W. S. Lawsor, J. Comp. Phys. 80, 253 (1989).

${ }^{15}$ M. Malacarne et al., Plasma Physics and Controlled Fusion 29, 1675 (1987).

16 P. C. Liewer, Nuclear Fusion 25, 543 (1985).

17 J. D. Callen, B. A. Carreras, P. H. Diamond, M. E. Benchikh-Lehocine, L. Garcia and H. R. Hicks, Plasma Physics and Controlled Nuclear Fusion Reseanch 1982, Proceedings of the 9th International Conference, Baltimore (IAEA, Vienna, 1983), vol. 1, p. 297. 

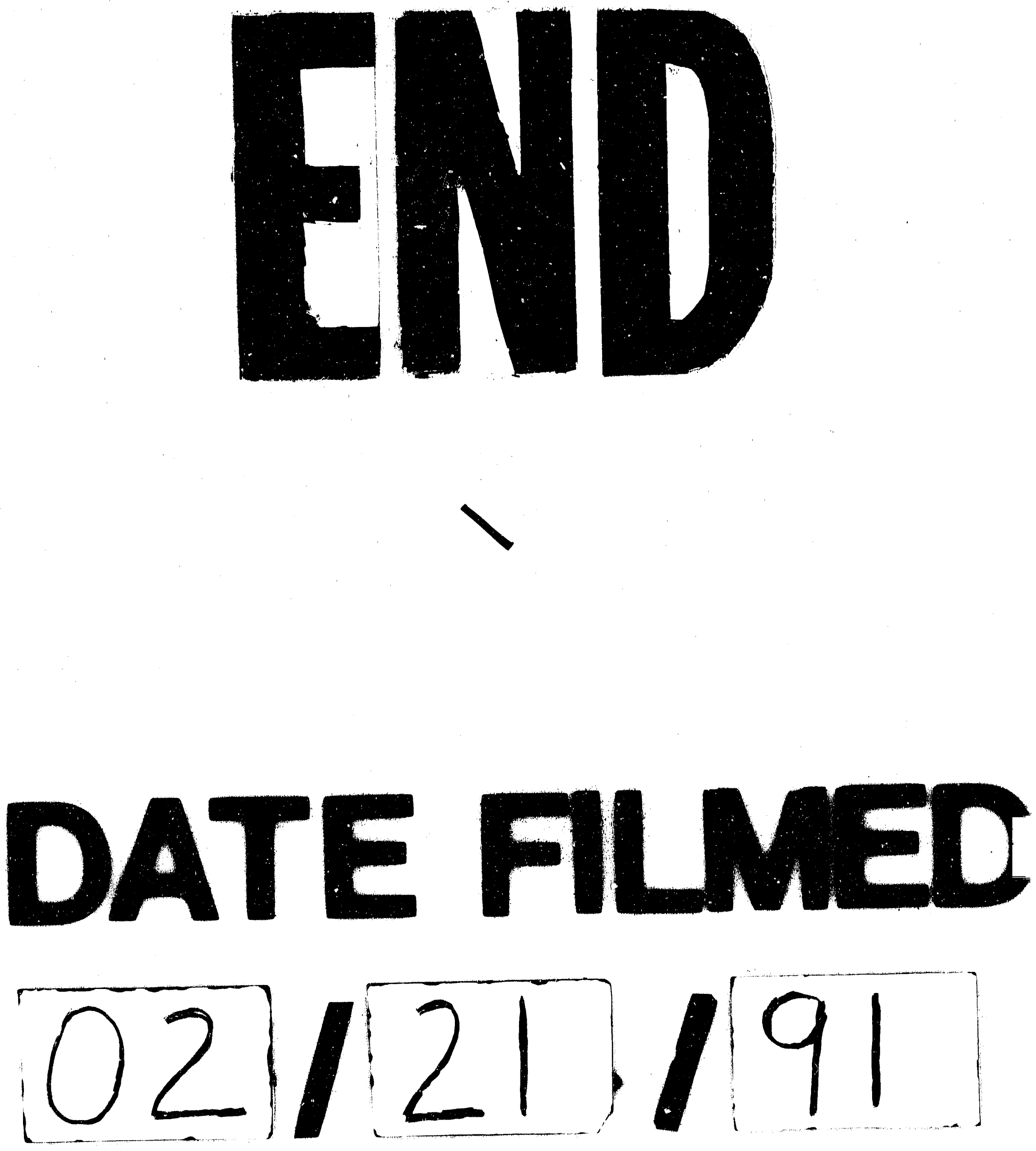
